Revue des patrimoines

La reconversion des sites et des bâtiments industriels

\title{
Interpréter les chais : le " grand îlot » des Chartrons à Bordeaux
}

\section{Chantal Callais}

\section{(2) OpenEdition}

\section{Journals}

Édition électronique

URL : http://journals.openedition.org/insitu/11883

DOI : $10.4000 /$ insitu. 11883

ISSN : 1630-7305

Éditeur

Ministère de la Culture

Référence électronique

Chantal Callais, «Interpréter les chais : le " grand îlot » des Chartrons à Bordeaux », In Situ [En ligne]

26 | 2015, mis en ligne le 07 juillet 2015, consulté le 25 mars 2020. URL : http://

journals.openedition.org/insitu/11883; DOI : https://doi.org/10.4000/insitu.11883

Ce document a été généré automatiquement le 25 mars 2020

\section{(c) (i) (9)}

In Situ Revues des patrimoines est mis à disposition selon les termes de la licence Creative Commons Attribution - Pas d'Utilisation Commerciale - Pas de Modification 4.0 International. 


\title{
Interpréter les chais : le « grand îlot » des Chartrons à Bordeaux
}

\author{
Chantal Callais
}

1 Le quartier des Chartrons s'est développé le long de la Garonne, au nord de la ville de Bordeaux, à travers une typologie singulière propre à l'activité du négoce du vin, combinant lieux de travail et habitations des négociants. Port et commerce ont quitté peu à peu ce site, laissant le quartier en grande partie abandonné au cours du $\mathrm{XX}^{\mathrm{e}}$ siècle. Depuis le milieu des années 1980, une vie nouvelle commence à investir cette friche industrielle urbaine très dense, d'abord de façon sporadique, par la reconversion de chais en habitations. Une première opération naît d'une initiative privée (rue Maurice) faisant appel à l'architecte Bernard Bühler. Elle sera suivie de plusieurs opérations d'habitat social avec le même architecte et de reconversions d'entrepôts en habitations d'initiative privée. En 1994, la ZAC (zone d'aménagement concerté) des Chartrons a pour ambition de revitaliser un vaste îlot du quartier en déshérence, le " grand îlot ». Combinant les objectifs de la ville et ceux de l'aménageur qui avait acquis une grande partie des entrepôts et immeubles de cet îlot, un projet de renouvellement urbain a émergé du génie du lieu. Fondé sur la spécificité de la structure foncière et sur le dialogue entretenu entre le patrimoine bâti et les édifices nouveaux, le nouveau quartier a été terminé en 2010. Mixité sociale et mixité fonctionnelle caractérisent la démarche et le résultat que l'on peut considérer aujourd'hui avec un certain recul.

\section{Un patrimoine singulier}

2 Le quartier des Chartrons s'est développé à partir du XIVe siècle. Les bourgeois de Bordeaux, alors sous domination anglaise, avaient obtenu des privilèges qui autorisaient leur seule production viticole à transiter gratuitement par la ville. Les autres stockaient leur marchandise hors les murs, le long des rives de la Garonne, aux Chartrons, qui doivent leur nom à l'installation des Chartreux en 1377. Au cours du temps, les négociants, étrangers, mais aussi bordelais, vont confirmer sa vocation commerciale et portuaire, et s'y retrouvent presque tous à la fin du XVIII ${ }^{e}$ siècle (fig. 1). 
Figure 1

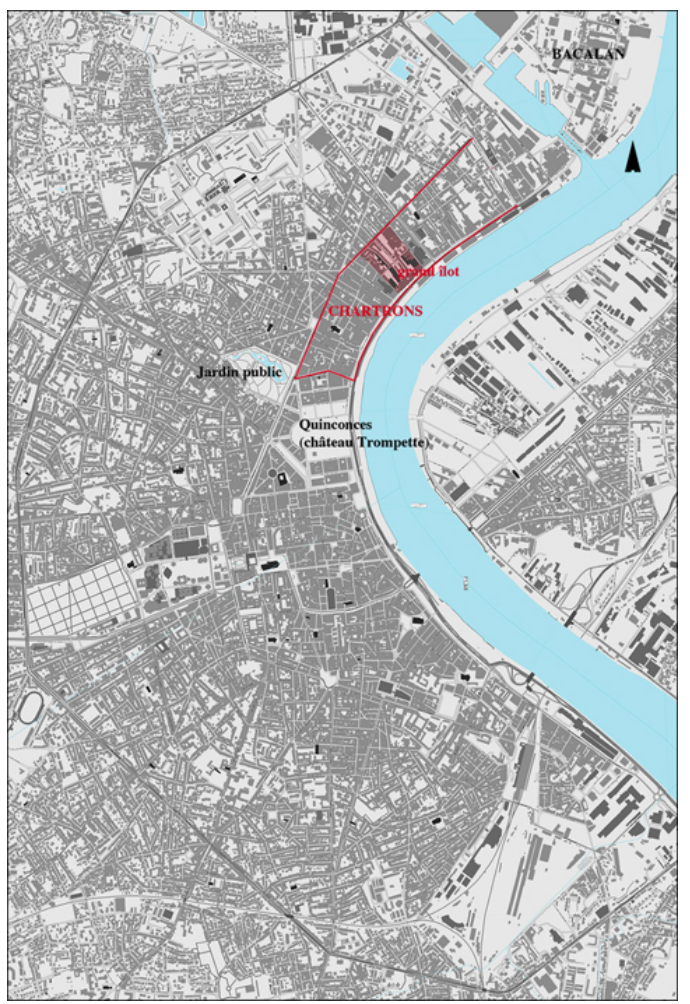

Situation du « grand îlot » au nord de la ville.

(c) Chantal Callais sur plan Géoportail.

Le château Trompette et son glacis isolent alors les Chartrons de la ville-centre. Bâti par Charles VII pour contrôler Bordeaux qu'il vient de reprendre à l'Angleterre (1451), la forteresse est reconstruite et la zone militaire inconstructible encore élargie par Louis XIV. Il faut attendre les grands travaux de l'intendant Tourny au milieu du XVIII ${ }^{e}$ siècle pour que le quartier se soude à la ville, relié et articulé au centre de Bordeaux et au faubourg de Saint-Seurin par le système des cours du «tour de ville » et le nouveau jardin public. Le lotissement des terrains du château Trompette (autour de la place des Quinconces) au début du XIX ${ }^{\mathrm{e}}$ siècle finalise le rattachement du quartier négociant à la ville.

4 Le rythme de développement des Chartrons suit celui du commerce : ralenti à la fin du $\mathrm{XVI}^{\mathrm{e}}$ siècle, il atteint son apogée pendant le brillant XVIII ${ }^{e}$ siècle bordelais. Limité à l'ouest par l'ancien chemin du Roi, il est irrigué au cours du XVII ${ }^{e}$ siècle par plusieurs voies nouvelles. La rue Notre-Dame (ancienne rue Saint-Joseph) traverse le quartier parallèlement aux quais et bute sur ce qui restera «le grand îlot». Plusieurs voies perpendiculaires la relient aux quais et au chemin du Roi (fig. 2). 
Figure 2

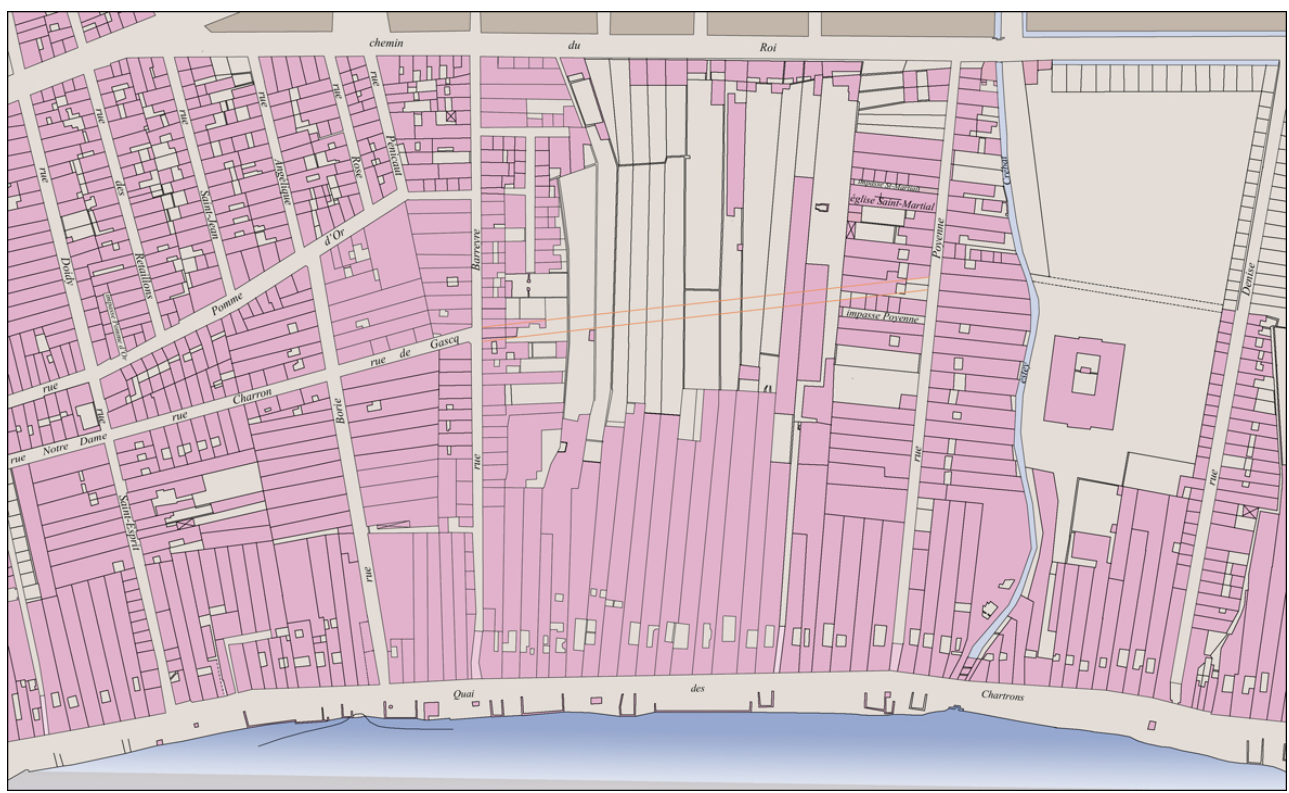

Plan cadastral de 1820-1830, indiquant le tracé du prolongement prévu de la rue Notre-Dame.

(c) Chantal Callais, d'après le cadastre des Archives municipales de Bordeaux.

5 À l'origine, les constructions sont de simples chais en bordure des quais, en avant de terres marécageuses occupées par des vignes ou des prairies. Jusqu'au XVI ${ }^{\mathrm{e}}$ siècle, si une habitation accompagne le chai, c'est dans le même volume. Les gravures du XVII siècle décrivent des chais à pignon sur rue, de un à deux niveaux, serrés le long des quais (fig. 3).

Figure 3

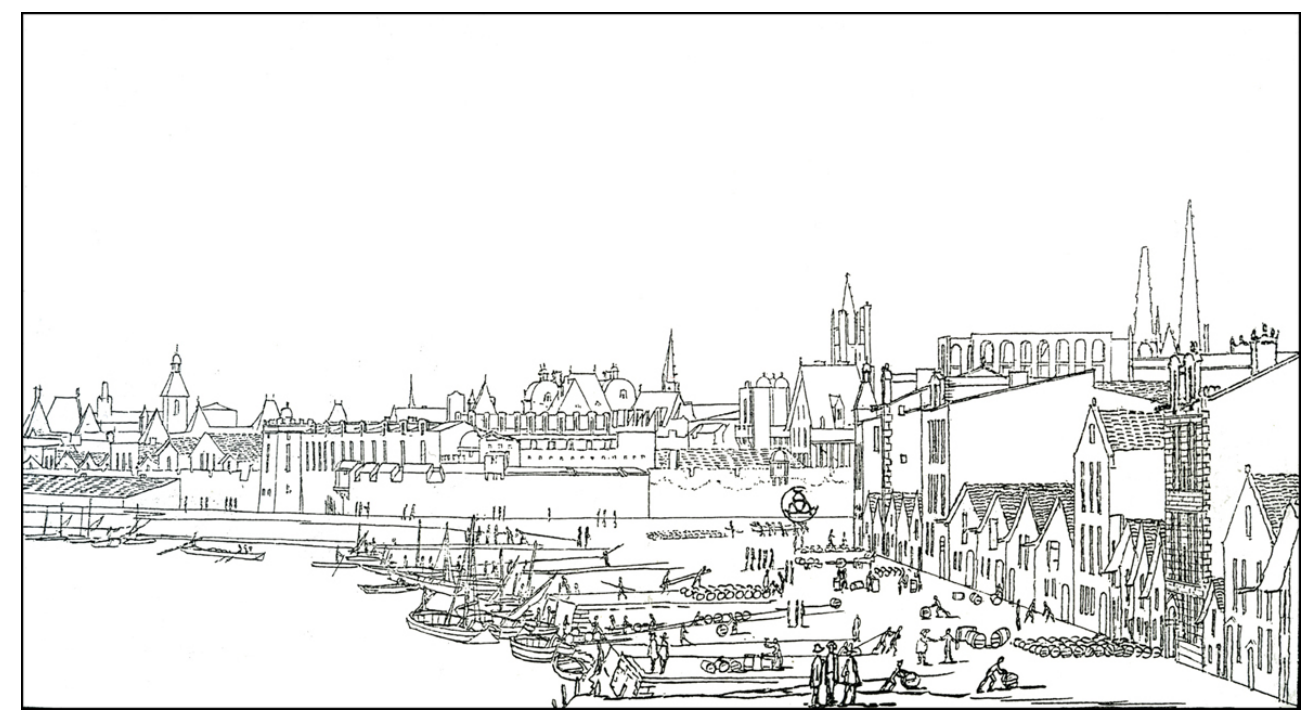

Quais aux abords du Château-Trompette, dessin à la plume de H. van der Hem, bib. Impér. de Vienne. (c) Archives historiques du département de la Gironde, Bordeaux et la région du sud-ouest, Société des archives historiques de la Gironde, 1904.

6 À partir de la seconde moitié du XVII ${ }^{\mathrm{e}}$ siècle $(1667)^{1}$, il est interdit de construire pignon sur rue. Entre 1750 et 1795 , la plupart des maisons ont été modernisées ${ }^{2}$ ou 
reconstruites selon un type qui est alors arrêté dans ses caractères permanents, combinant les parties nobles - les appartements et les bureaux des négociants - et la partie «travail» avec les chais. En 1770, l'historien Dom Devienne considère que la "façade des Chartrons est aujourd'hui plus considérable que celle de la ville ${ }^{3}$. Moins régulière que la grande façade à programme de Tourny au sud, elle en constitue en effet un pendant plus capricieux, mais encore très monumental. Depuis 1741, la grande majorité des maisons présente sur le quai leur mur gouttereau (seules subsistent avec pignons sur rue les fameuses «maisons hollandaises ", qui furent en réalité bâties par Hilaire Renu, marchand de Bordeaux en 1679-16804) (fig. 4). La continuité quasi absolue du front bâti est préservée grâce au dispositif d'immeubles-porches sous lesquels passent la majorité des rues perpendiculaires aux quais.

Figure 4

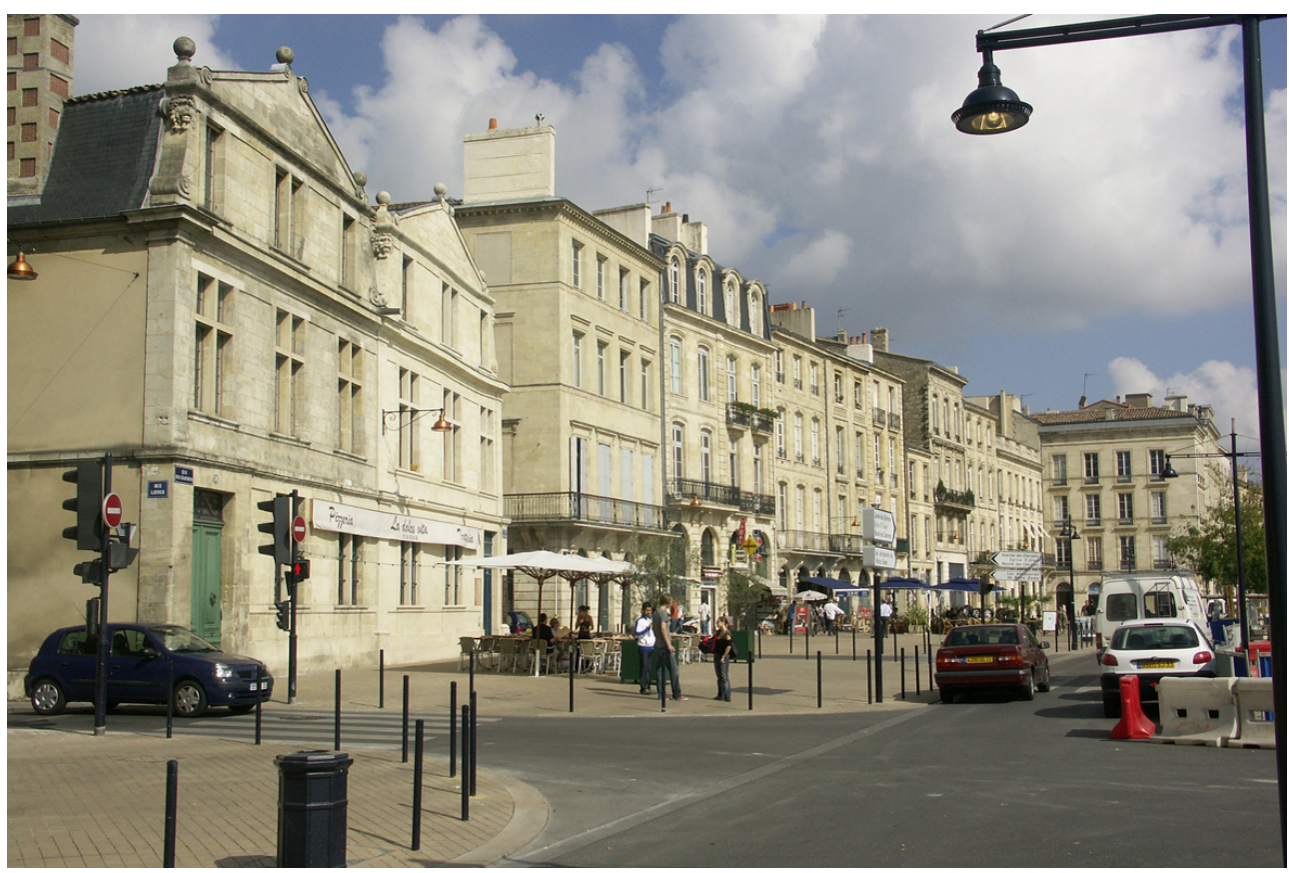

Les « maisons hollandaises ».

Phot. Callais, Chantal. (c) Chantal Callais.

7 Le type de la maison à chai des négociants se précise et se définit jusqu'à être celui systématiquement construit dans le quartier au cours des XVIII ${ }^{\mathrm{e}}$ et $\mathrm{XIX}^{\mathrm{e}}$ siècles. Il occupe une parcelle de 10 mètres de largeur environ sur une profondeur habituelle de 75 à 140 mètres. Comme l'immeuble bourgeois couramment construit en centre-ville, il se compose de deux corps de bâtiments en pierre : le premier, le plus noble, de trois ou quatre niveaux, profond de 13 à 20 mètres, est séparé par une cour égale à la profondeur du second moins épais et en général moins haut, exempt de décor (fig. 5). À ce double immeuble succède le chai, par l'intermédiaire d'une seconde cour souvent couverte sur un niveau. Il semble que l'origine du mot soit gauloise, le «caio ${ }^{5} »$. Très protégé par un plafond très épais en multicouches pour en garantir l'isothermie, c'est le lieu où sont entreposés les vins, en barriques, tonneaux, cuves ou bouteilles et qui peut intégrer une tonnellerie. 


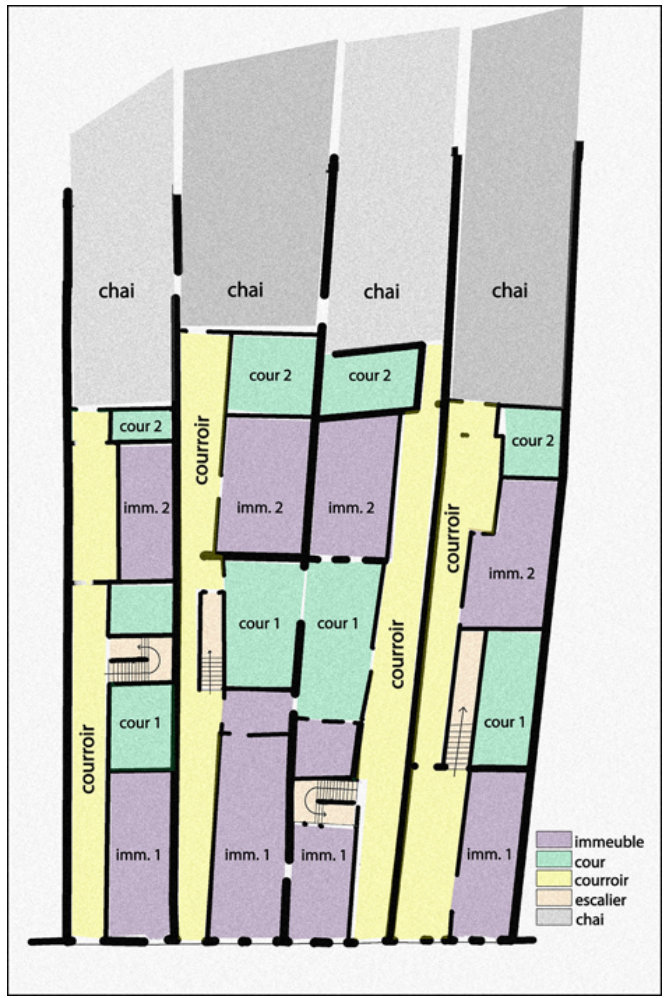

Plan schématique des parcelles de Luze (les chais sont coupés)

(c) Ch. Callais d'après le dessin établi par A. Liquard, op. cit.

8 Un passage latéral, appelé d'abord corridor, puis « courroir » ou « courroire ", distribue à travers plusieurs séquences les bureaux du rez-de-chaussée, la cour, l'escalier, puis le second bâtiment pour mener finalement au chai qui s'étend à l'arrière, après quelquefois une seconde cour. Il n'y a donc qu'une seule entrée pour accéder au lieu de travail et à l'habitation qui s'organise dans les étages. Le courroir se compose de trois séquences. L'entrée est la partie la plus noble, puis le passage se rétrécit pour donner accès à la cour et à la cage d'escalier. La troisième séquence, qui peut être aussi large qu'une pièce, distribue les pièces du rez-de-chaussée du second immeuble (salle d'expédition de lavage, rinçage et embouteillage et plus récemment vestiaires ou pièce de restauration) et donne accès au chai. 
Figure 6

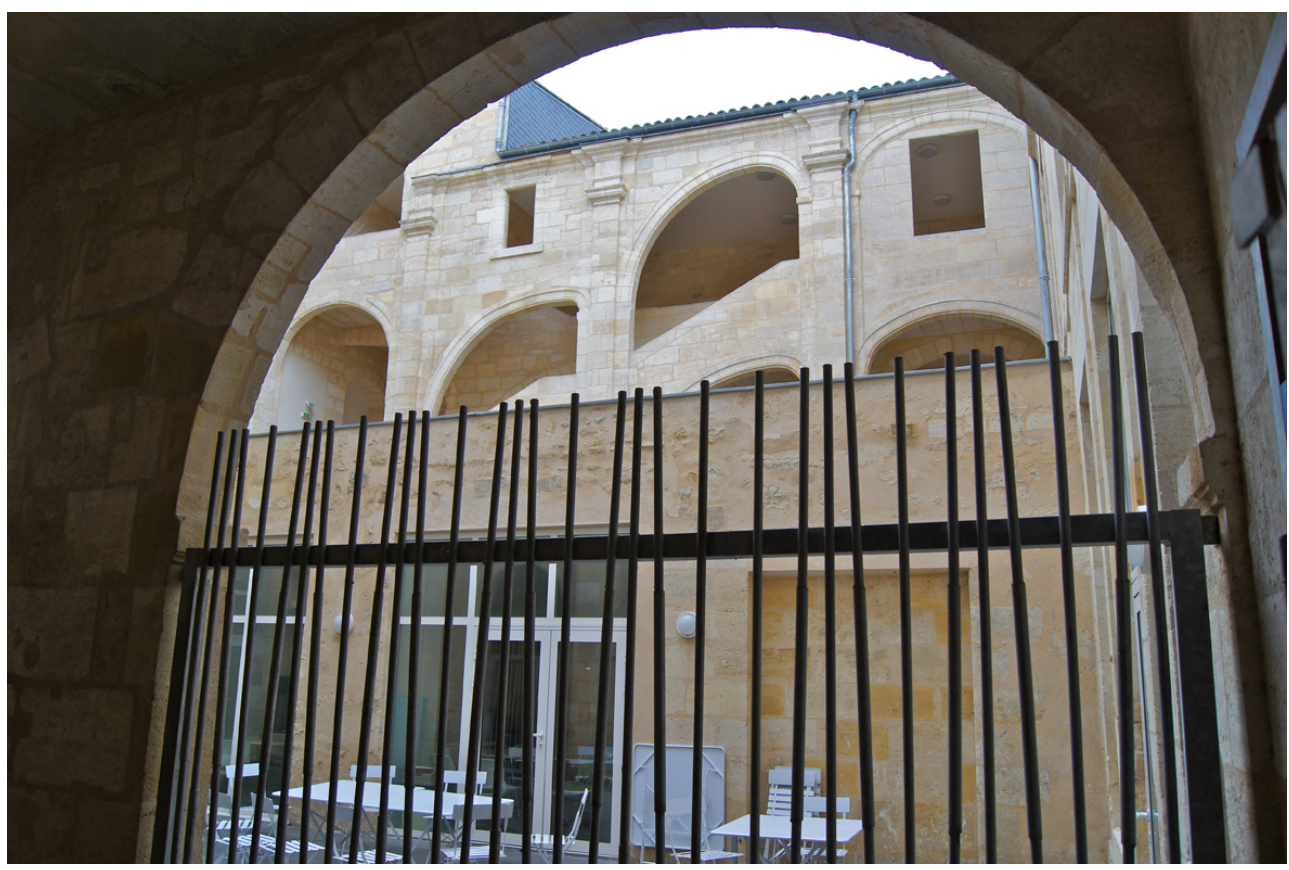

Vue de l'escalier du n`88 depuis le courroir (la galerie 89) du n89 quais des Chartrons.

Phot. Callais, Chantal. (c) Chantal Callais.

9 Il arrive souvent que les cours soient couvertes sur un ou deux niveaux, traitées alors en puits de jour. L'escalier, à une ou deux volées droites (fig. 6), perpendiculaire ou parallèle aux mitoyens, est toujours unique. Il peut être situé dans la cour, ou dans l'un ou l'autre des immeubles et relié à une galerie pour desservir l'ensemble ; il est éclairé par une verrière zénithale ou par des ouvertures sur la cour, comme on le fait depuis le XVII ${ }^{e}$ siècle dans les immeubles à deux corps. Le caractère particulier de ce dispositif est le détachement de la cage d'escalier du mur mitoyen du côté du courroir, qui permet ainsi une distribution de tous les éléments qui composent l'ensemble indépendamment les uns des autres (la cour n'est jamais distributrice et ne donne jamais accès à la cage d'escalier). Avec le temps, les fonctions d'habitation et de commerce se sont souvent dissociées, aboutissant à la création d'un couloir doublant le courroir qui devient alors l'accès exclusif au chai. Le dispositif du courroir, de 1, 80 à 4, 50 mètres de largeur, présente aujourd'hui l'intérêt de pouvoir facilement traverser un immeuble à la façon d'un passage cocher et de préserver une certaine perméabilité de l'intérieur de l'îlot si elle est souhaitée (fig. 7). 
Figure 7

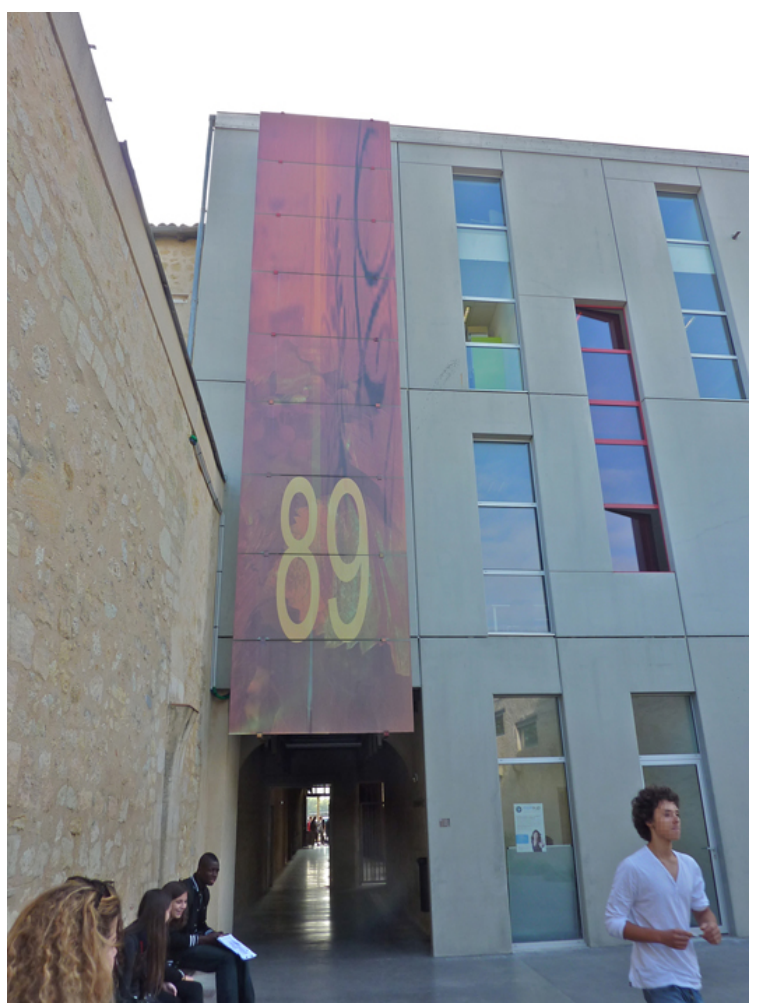

Entrée dans la galerie 89.

Phot. Callais, Chantal. (c) Chantal Callais. de 100 mètres environ. Elle s'étend peu à peu sur trois parcelles, les $n^{\text {os }} 88,89$ et 90 , construits entre 1795 et 1810. Sur le chemin du Roi (futur cours Balguerie-Stuttenberg), un autre propriétaire s'installe de la même façon (chai Lacaze, au ${ }^{\circ}{ }^{52}$ ). Un grand jardin demeure encore au centre de l'îlot en 1890, propriété d'Albert de Luze depuis $1850^{6}$. Le prolongement de la rue Notre-Dame apparaît en projet sur les plans cadastraux de 1830 et de 1850 . Mais ce projet ne sera jamais mis en œuvre et la rue butera toujours sur ce qui s'affirme comme le "grand îlot", tandis que la rue SaintePhilomène est percée au-delà vers le nord, dans la continuité virtuelle de la rue NotreDame. Entre la fin du XVII siècle et 1940 , le chai de Luze sis au n ${ }^{\circ} 88$ ne cesse de s'étendre, jusqu'à annexer totalement la parcelle du cours Balguerie-Stuttenberg et atteindre alors 360 mètres de profondeur7. En 1966, la maison de Luze s'étend encore en acquérant le $n^{\circ} 87$ du quai des Chartrons et le $n^{\circ} 19$ rue Barreyre, y gagnant un troisième accès sur lequel ouvre un agrandissement de la salle d'embouteillage construit en charpente métallique.

11 Le grand îlot, compris entre les quais à l'est, le cours Balguerie-Stuttenberg à l'ouest, les rues Barreyre et Poyenne au sud et au nord, est ainsi resté indivisé et quasiment indivisible. Le projet de prolongement de la rue Notre-Dame, qui apparaît dans les projets et sur les plans cadastraux du $\mathrm{XIX}^{\mathrm{e}}$ siècle (voir fig. 2), est repris plus récemment par l'agence d'urbanisme, qui abandonne l'idée en $1985^{\circ}$. Il faut attendre le début des années 1990 pour que ce territoire non occupé en pleine ville fasse l'objet d'un projet de renouvellement. 


\section{Un projet urbain : une démarche nouvelle à Bordeaux} œuvre des démarches qui amorcent le réveil de la ville alors qualifiée de «belle endormie ». Les consultations et expertises qu'il lance en 1991 aboutissent l'année suivante à la nomination de Dominique Perrault comme concepteur, puis de Serge Goldberg comme délégué à l'Aménagement et au développement de la ville. Celui-ci, polytechnicien et urbaniste, ancien directeur de l'EPA (Établissement public d'aménagement) de la ville nouvelle de Saint-Quentin-en-Yvelines, alerte rapidement la ville sur le potentiel de renouvellement que représente le "grand îlot » du quartier des Chartrons, d'une surface double des îlots habituels du quartier, avec des parcelles de 100 à 350 mètres de longueur. La désaffection des chais le laissait pratiquement vide d'occupation, ce que le bailleur social et aménageur Domofrance avait déjà repéré, en se rendant acquéreur de plusieurs parcelles. Jean-Luc Hoguet, alors directeur de l'office, explique que la décision d'acheter ces parcelles alors peu onéreuses relevait un peu d'un pari pour un aménageur social, mais qu'il avait été rapidement convaincu de l'opportunité que représentait ce site au cœur de la ville et de la nécessité de maîtriser suffisamment de foncier pour permettre un véritable projet d'ensemble cohérent ${ }^{9}$.

En 1993, la Ville prend l'initiative d'organiser un concours sur l'aménagement des cinq hectares renouvelables de l'îlot. L'architecte et urbaniste Alain Charrier (de l'agence d'architecture $\mathrm{BBCR}$ ), associé à cette occasion avec l'historien Robert Coustet, en est le lauréat. La commande du concours est en elle-même intéressante. Le programme était celui d'un quartier de ville, essentiellement composé de logements de statuts et de types variés (social et privé, collectif et individuel) et d'équipements d'usage quotidien (une école, un gymnase). La question était surtout de proposer un mode d'organisation de ce futur nouveau quartier, introduisant à Bordeaux la notion de "projet urbain ", au sens formel du terme. Bien après Paris et quelques autres villes en France, le projet des Chartrons est ainsi le premier véritable "projet urbain " contemporain à Bordeaux, dans la mesure où le projet à l'échelle urbaine est dissocié dans le temps des projets d'architecture qui composeront le quartier. La démarche se rapproche des processus de fabrication de la ville historique et s'éloigne de la pratique de "plan de masse » qui a dominé au $\mathrm{XX}^{\mathrm{e}}$ siècle en combinant de façon figée dans un même projet les deux échelles. À l'appui du principe, l'architecte concepteur du projet du quartier reçoit une mission de maîtrise d'œuvre urbaine pour assurer le suivi et la coordination sur la durée de la construction des immeubles dessinés par différents architectes.

Domofrance, propriétaire de la moitié de la surface de ce nouveau quartier, adhère rapidement aux principes du projet lauréat du concours. Une convention est établie entre la Ville, la Communauté urbaine (CUB) qui a les ZAC dans ses compétences, et Domofrance qui reçoit la délégation d'aménagement. Société de logement social ouverte aux expérimentations et consciente de la valeur pédagogique de la démarche, Domofrance va s'investir avec enthousiasme en tant que maitre d'ouvrage aménageur dans la mise en place du projet. Aux différents stades de l'avancement de l'opération, l'office mène des actions destinées à informer les habitants du quartier, les étudiants en architecture ou en urbanisme et tous les publics intéressés par le renouvellement du grand îlot. Le support principal en est une exposition qui évolue avec l'avancement du projet. Intégrée dans le grand projet urbain de 1996 du nouveau maire Alain Juppé (élu en 1995), la ZAC du renouvellement du grand îlot des Chartrons est votée par la CUB en 
1998. Sur une surface totale de neuf hectares, la partie opérationnelle en comprend quatre et demi. Le dernier immeuble de la ZAC a été livré en 2010, clôturant la douzaine d'années que la construction de ce quartier a nécessitée (les premières opérations ont commencé en 1999).

\section{Une forme urbaine inscrite dans une histoire}

Même orphelines des activités qui ont justifié leur construction, les formes et les espaces demeurent ${ }^{10}$. Ils ont un rôle dans l'identification de la ville et une autonomie relative par rapport aux fonctions qu'ils accueillent. Nés du commerce du vin et de la nécessité de son stockage qui justifient leur disposition, les chais vides de leur occupation marquent encore la structure spatiale des Chartrons. Le territoire du projet du grand îlot des Chartrons était construit à plus de $85 \%$ et très hermétique. La partie opérationnelle de l'îlot en exclut certaines zones occupées, notamment au nord-ouest, par les archives départementales à l'angle du cours Balguerie-Stuttenberg et de la rue Poyenne et un petit ensemble de maisons distribué par un dispositif en impasse à l'angle du même cours et de la rue Barreyre au sud-ouest. À l'est, le front bâti sur le quai des Chartrons est protégé et doit être conservé intégralement. L'option de la tabula rasa est d'emblée écartée. Alain Charrier affirme en 1995 :

L'histoire nous lègue un patrimoine. À nous de faire preuve d'invention et d'ingéniosité pour savoir le réutiliser et le réintégrer dans une nouvelle « histoire urbaine ». Cela permettra sans nul doute de concevoir de nouvelles typologies (par exemple de logement) et de nouveaux modèles urbains. L'addition des contraintes du site est pour nous un élément de liberté créatrice ${ }^{11}$.

Ainsi, sa démarche a consisté à se nourrir du génie du lieu pour fonder le projet. On peut distinguer deux phases dans la mise en place des principes de l'organisation spatiale: la première établit les moyens d'organiser la pénétration de l'îlot et ses liaisons au quartier, la seconde propose d'appuyer la structure spatiale du futur quartier sur son organisation foncière historique ${ }^{12}$.

\section{Tisser la ville : irriguer le grand îlot}

17 Pour irriguer ce territoire, une solution, suggérée par le projet ancien de la ville, consistait à poursuivre la rue Notre-Dame pour rejoindre la rue Sainte-Philomène, selon le tracé qui transparaît sur les plans cadastraux de 1820 et de 1850. Le grand îlot ayant échappé historiquement à cette logique de découpage, Alain Charrier a pensé qu'il était intéressant de conserver partiellement cet aspect exceptionnel et introverti du site et d'imaginer d'autres solutions pour la création des nouveaux espaces publics. Organisé autour d'une place nouvelle au centre de l'opération, le nouveau quartier est relié au réseau principal de la ville par un mail qui s'ouvre largement sur le cours Balguerie-Stuttenberg, seule artère importante sur laquelle il était possible d'ouvrir largement, en suivant le tracé d'une parcelle de chais deux fois plus large que les autres. Le mail (allée des Vignes, devenue depuis allée Stendhal) et la place (place des Vignes au moment du projet, devenue place Paul-et-Jean-Paul-Avisseau) se réfèrent aux espaces publics du quartier des Chartrons. L'architecte a étudié les places qui ponctuent les Chartrons (la place Paul-Doumer, la place du marché des Chartrons, la place Saint-Martial), leur dimensionnement et leurs relations au tissu urbain voisin. Il en montre la diversité, mais aussi les similitudes dimensionnelles. Des documents de 
travail expliquent la démarche: la nouvelle place est installée sur un schéma d'ensemble du quartier et son plan est superposé à celui des autres places pour mettre en évidence les rapports d'échelle et les liens au contexte urbain (fig. 8).

Figure 8


Dessins des archives d'Alain Charrier, dans É. Martin. Le patrimoine au service de la modernité

(C) Agence BBCR.

La nouvelle place combine le caractère protégé de la place du marché des Chartrons avec un accès monumental au cours Balguerie-Stuttenberg par un mail évoquant les caractéristiques du cours Xavier-Arnozan, voie majeure et prestigieuse de ce quartier de négociants, dont plusieurs y mettent en scène leurs demeures à travers une architecture à programme à la fin du XVIIIe siècle. Les dimensions du nouveau mail, l'allée Stendhal, sont un peu réduites par rapport au modèle (22 mètres au lieu de 35 mètres), et surtout son terre-plein central n'est que partiel, la plus grande partie étant réservée à des places de stationnement privées. Des rues secondaires relient la place au sud et au nord aux rues Barreyre et Poyenne. Ici encore, l'architecte s'en rapporte à la tradition des voies sous porche qui sont un facteur identifiant du quai des Chartrons et lui permettent d'insister sur l'ambiance protégée qu'il souhaite pour ce nouvel espace public (fig. 9). 
Figure 9

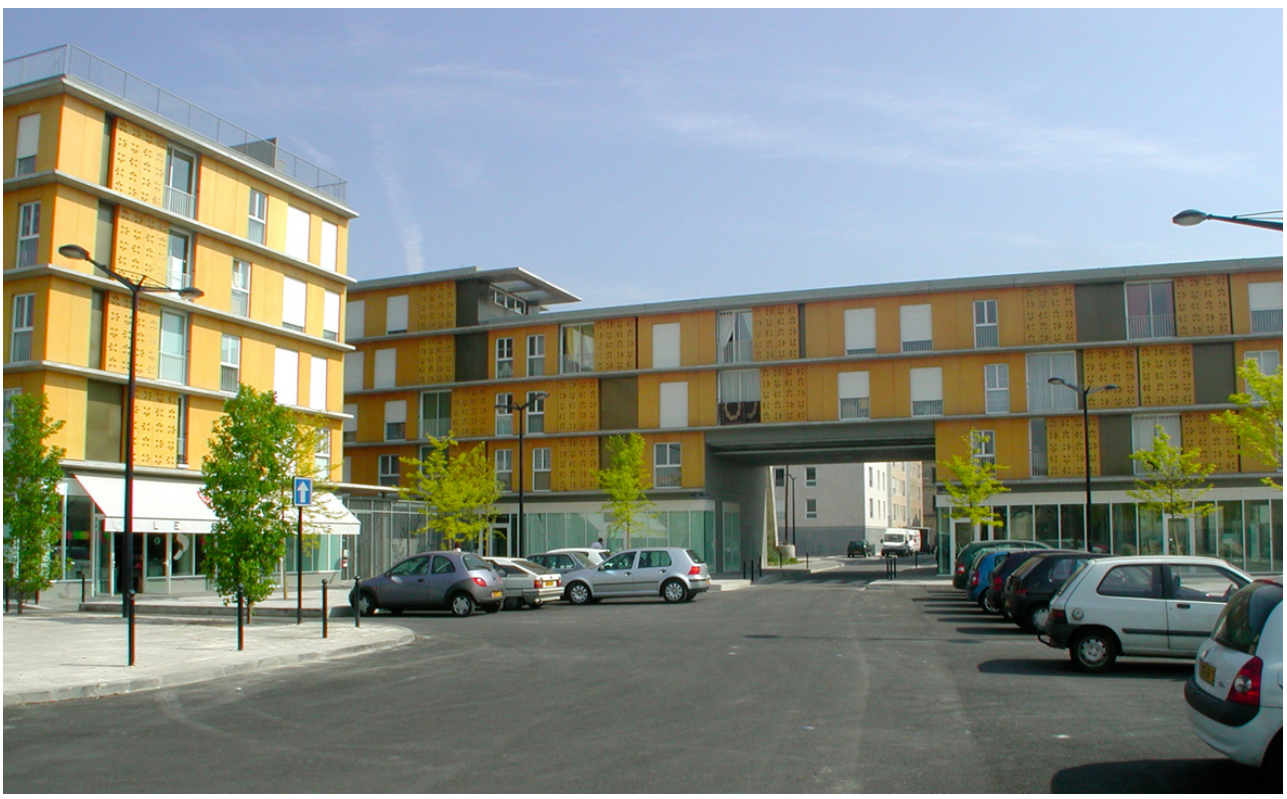

L'immeuble-pont (BLP architectes) donnant accès à la place au nord.

Phot. Callais, Chantal. (c) Chantal Callais.

19 Le dernier lien avec le réseau viaire existant est une rue parallèle au mail, ouvrant sur le cours Balguerie-Stuttenberg (la rue des Chais, devenue rue du Faubourg-des-Arts) qui correspond au ruban d'un chai détruit et annonce le second principe fondateur de la forme urbaine du nouveau quartier, la conservation de la trame parcellaire. Un des dessins d'étude suggérait de percer d'autres rues destinées à relier la rue Barreyre à la rue du Faubourg-des-Arts en désenclavant le petit groupe de maisons à l'angle sudouest de l'îlot et enfin de mettre en scène la rue Notre-Dame à son aboutissement sur le grand îlot par une placette qui donnait un peu de souffle à l'étroite rue Barreyre (fig. 10). Le projet final laisse cette possibilité ouverte, mais elle ne sera pas mise en œuvre (les tracés des rues sont signalés en pointillés sur le plan d'aménagement de zone, mais hors périmètre de la ZAC, par non-maîtrise du foncier). 
Figure 10

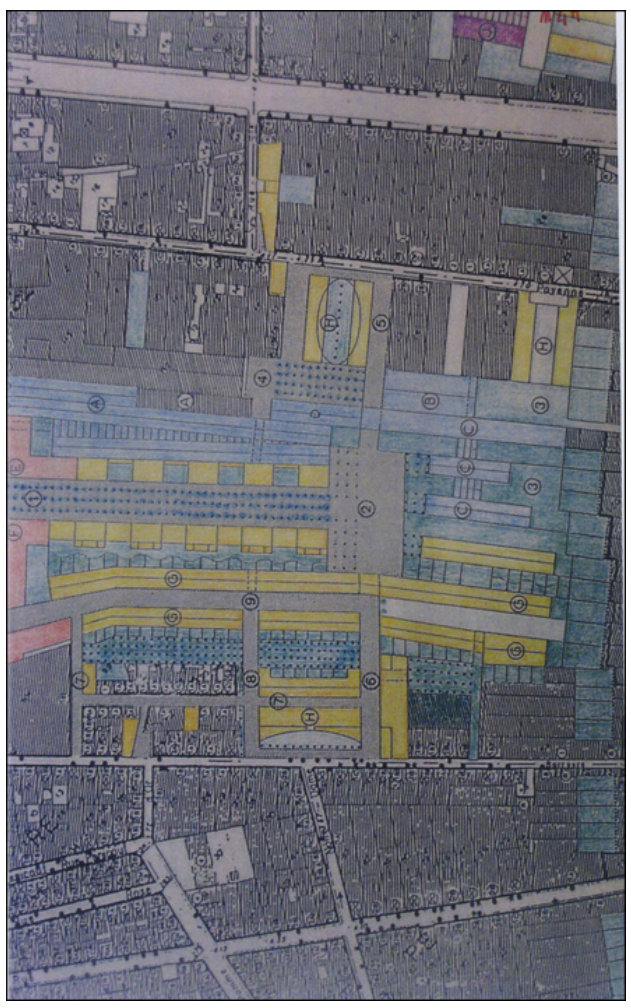

Étude du plan de la ZAC. Archives A. Charrier dans É. Martin. Le patrimoine au service de la modernité. (c) Agence BBCR.

\section{La structure foncière comme patrimoine identitaire}

La structure parcellaire singulière du quartier, en longues lanières d'une dizaine de mètres de largeur, apparaît rapidement aux concepteurs comme le caractère fondamental du quartier, susceptible de l'inscrire dans une filiation historique identitaire. La conservation de cette structure foncière présentait l'intérêt de faire de cette opération de renouvellement urbain un projet « situé » et d'induire un paysage et des types d'habitat qui seraient propres à ce lieu, comme le souligne Alain Charrier. Le choix est fait d'alterner une lanière bâtie et une lanière libre (fig. 11). 


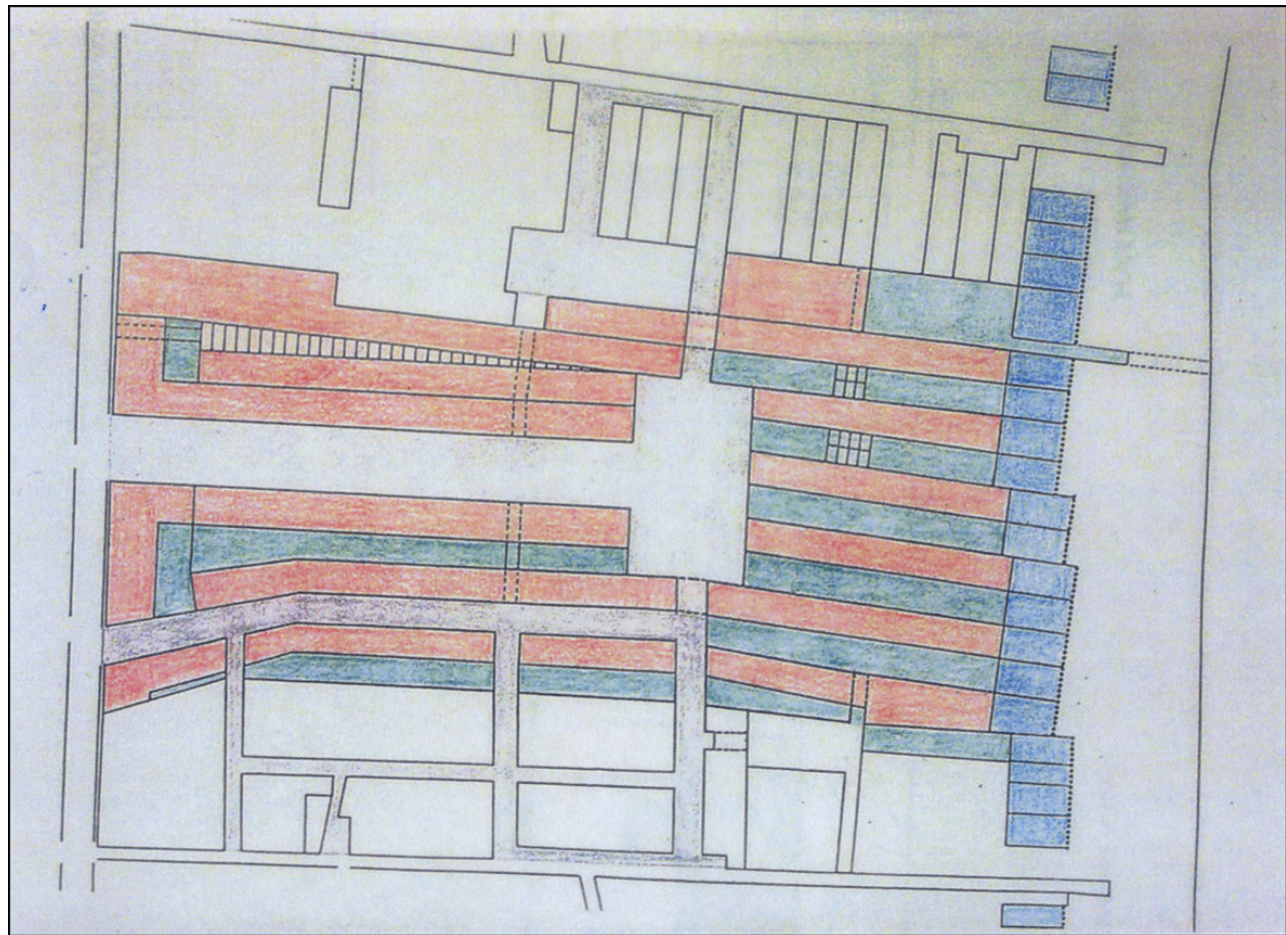

Étude du principe de l'alternance des lanières pleines et vides. Archives A. Charrier dans É. Martin. Le patrimoine au service de la modernité.

(c) Agence BBCR. dispositif autorise la préservation des plus belles constructions en pierre qui sont mêlées à des bâtiments contemporains et confortent le lien du nouveau quartier avec son passé. Les lanières vides peuvent devenir des rues, des passages, des jardins, des cours... L'architecte établit des schémas de principe pour démontrer la faisabilité du concept sur cinq ou sept travées d'épaisseur, pour des programmes d'habitations individuelles (fig. 12).

\section{Figure 12}



Étude de faisabilité de la reconversion des chais. Archives A. Charrier dans É. Martin. Le patrimoine au service de la modernité.

(c) Agence BBCR.

L'adaptation du principe à la réalité du terrain va considérablement l'enrichir, grâce aux contraintes de situation de chaque « lanière » et grâce à la combinaison des chais 
conservés avec les bâtiments nouveaux. Il était nécessaire également de pouvoir densifier en hauteur à certains endroits pour que l'opération soit économiquement viable.

\section{Le plan d'aménagement : un assemblage de parcelles et des îlots perméables}

Le plan d'aménagement de zone (PAZ) destiné à la mise en œuvre du projet, accompagné d'un règlement (RAZ), est volontairement simple (fig. 13). Il indique les espaces publics, les bâtiments à conserver, les alignements obligatoires et les implantations préconisées sur chaque parcelle opérationnelle. Ces lots à bâtir peuvent se composer d'une à six parcelles anciennes en épaisseur sur des longueurs plus ou moins importantes. Sauf les chais reconvertis qui conservent leur hauteur, d'autres bâtiments atteignent quatre à cinq étages. Des types d'assemblage de logements très variés s'esquissent clairement : logements en bandes, organisation autour de courées, immeuble à redents, immeubles-ponts. Si certains équipements sont situés à ce stade d'avancement du projet (l'école, le gymnase), d'autres hypothèses ont évolué avec le temps.

Figure 13

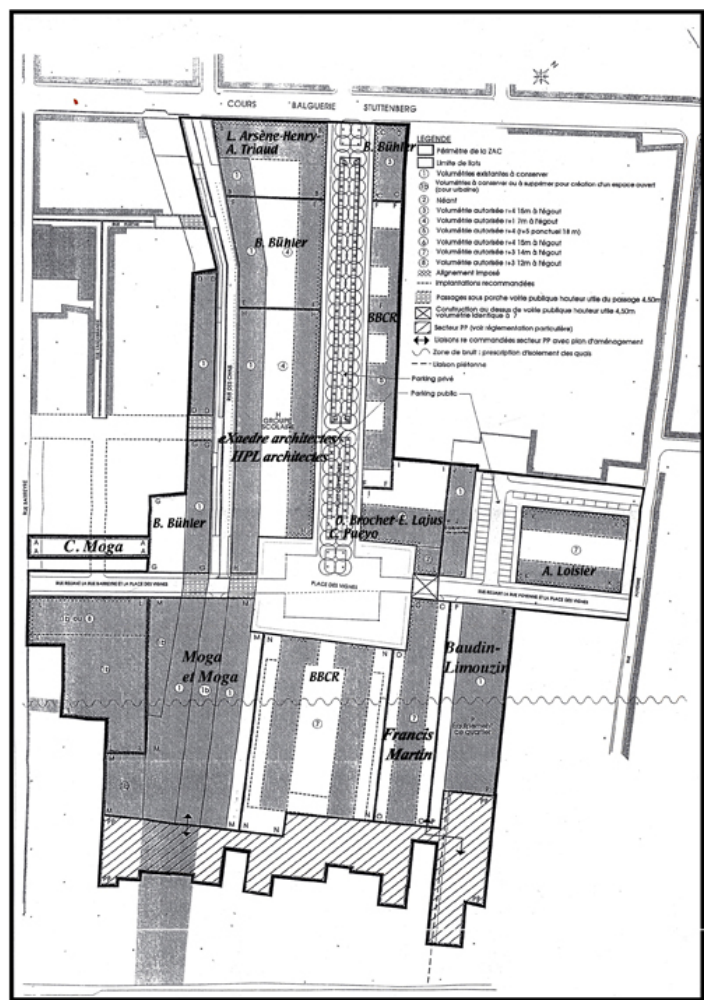

Plan d'aménagement de zone de la ZAC des Chartrons. Document A. Charrier.

(c) Agence BBCR.

La répartition des lots à construire est faite selon un principe parcellaire, résultant de la combinaison de plusieurs parcelles anciennes laissant lisible le dispositif en lanières. L'îlot n'est pas confondu avec l'unité opérationnelle, comme c'est souvent le cas dans des opérations contemporaines, mais il est le résultat de la somme des parcelles. 
L'alternance des lanières libres et construites, perceptible depuis les espaces publics, en fait des îlots perméables, soit par le regard, soit par des passages privés distributifs, qui peuvent parfois être ouverts au public (fig. 14).

Figure 14

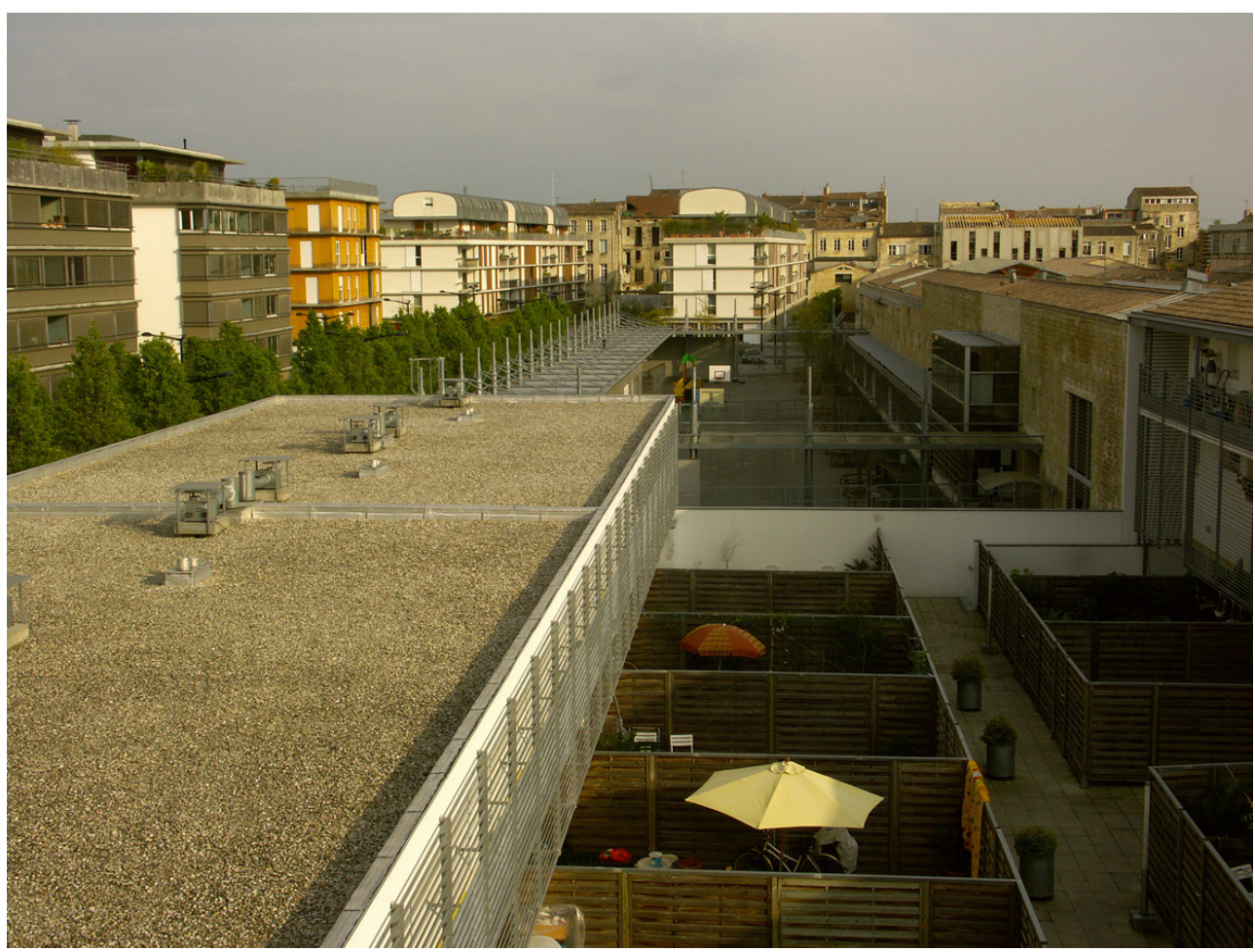

Vue de la ZAC depuis un point haut de la cour à l'arrière du cours Balguerie-Stuttenberg : au premier plan, les terrasses des maisons de ville (B. Bühler), puis l'école et sa cour. Au fond, dans l'axe du mail, l'«immeuble-square».

Phot. Callais, Chantal. (c) Chantal Callais.

Deux passages en particulier sont prévus pour permettre de relier le nouveau quartier aux quais des Chartrons. Empruntant le tracé des anciens courroirs, ils découlent quasiment naturellement de ce système distributif originel des constructions anciennes permettant de traverser la double croûte bâtie des quais dont les immeubles sont réhabilités en logements par Domofrance entre 1995 et 2000. À l'arrière de ces immeubles des quais est organisée une zone de stationnement. Plantée d'arbres de haute tige, elle forme l'interface de la partie nouvelle et de la partie réhabilitée des quais. En l'absence de voitures (soit provisoire, soit définitive), son aménagement en fait une sorte de jardin collectif ombragé appropriable par les habitants (fig. 15). 
Figure 15

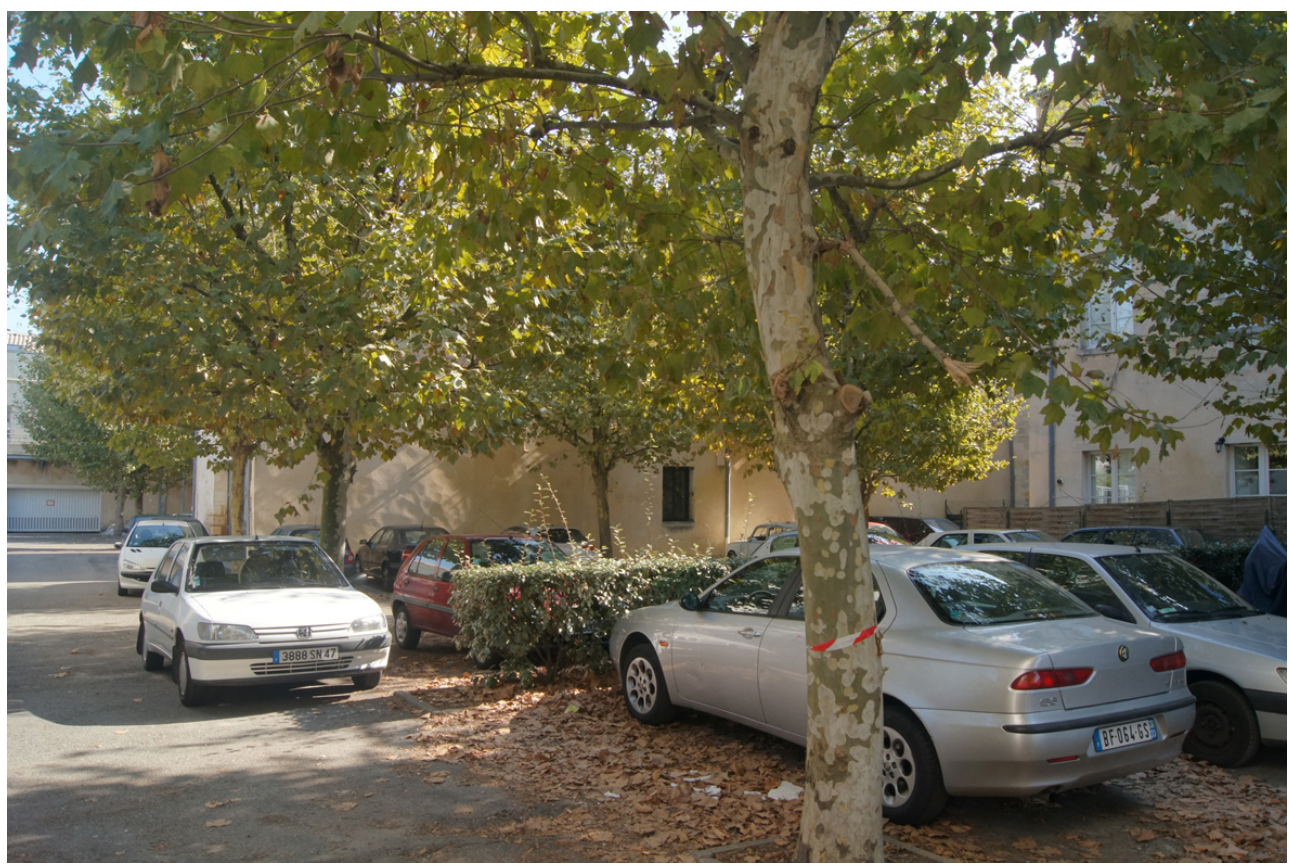

Zone « tampon » entre l'arrière des immeubles du quai des Chartrons et la ZAC, servant pour les stationnements des logements réhabilités dans les immeubles des quais.

Phot. Callais, Chantal. (c) Chantal Callais.

Le projet de la forme urbaine a permis de maîtriser le long temps que demande l'édification d'un morceau de ville de cette ampleur, avec suffisamment de précisions pour mettre en œuvre l'idée de ville projetée, mais avec assez de souplesse pour adapter avec le temps les affectations programmatiques et entrer en dialogue avec les propositions des différents architectes qui interviennent comme maîtres d'œuvre à l'échelle architecturale.

\section{Un projet fondé sur des dialectiques}

27 Le projet trouve ses qualités principales dans l'intelligence dialectique qu'il instaure entre différents enjeux : celle des choix des programmes et de leur emplacement, celle entre les deux échelles de projet, urbain et architectural, clairement identifiées et articulées, enfin celle qui s'établit dans les projets entre l'ancien conservé et les écritures contemporaines.

\section{De la forme aux fonctions}

L'organisation formelle proposée par l'architecte est mise en résonance avec les programmes fonctionnels. Ces programmes sont situés dans l'espace en fonction de la logique formelle du projet, des contraintes foncières ou des opportunités techniques. Les caractéristiques d'un vaste chai en construction métallique et d'une largeur exceptionnelle, sans qualité architecturale particulière, répondent bien au gabarit nécessité par un gymnase. Son emplacement étaye ce choix : potentiellement accessible par un passage depuis les quais, il sera facilement utilisable par les scolaires provenant de plusieurs écoles du quartier. Il est finalement entièrement reconstruit en respectant 
le gabarit initial par les architectes Baudin et Limouzin. L'école eXaedre architectes/ HPL architectes occupe trois parcelles au centre du nouveau quartier. Cette implantation, entre la place et le mail, répond bien aux pratiques de l'espace public liées à une école. Par ailleurs, comme il faut attendre la construction des logements et l'apport de population qu'ils supposent pour construire cet équipement, ce délai laisse le temps nécessaire à la complète maîtrise foncière de la parcelle concernée (fig. 16).

\section{Figure 16}



Cadastre actuel (d'après cadastre.gouv.fr) indiquant les différents programmes, les rues nouvelles (entourées en rouge), les passages (entourés en vert). Les programmes autres que le logement sont indiqués en orangé.

Repro. Callais, Chantal. (c) Chantal Callais (d'après cadastre.gouv.fr).

Il est prévu d'implanter des locaux commerciaux dans une partie des rez-de-chaussée, notamment sur le cours Balguerie-Stuttenberg et la place de quartier. Enfin, la volumétrie des chais voûtés se prête bien à l'installation d'atelier d'artistes et de leur boutique, en lien avec les activités de la proche rue Notre-Dame, bordée d'antiquaires et de galeries d'art (fig. 17). 
Figure 17

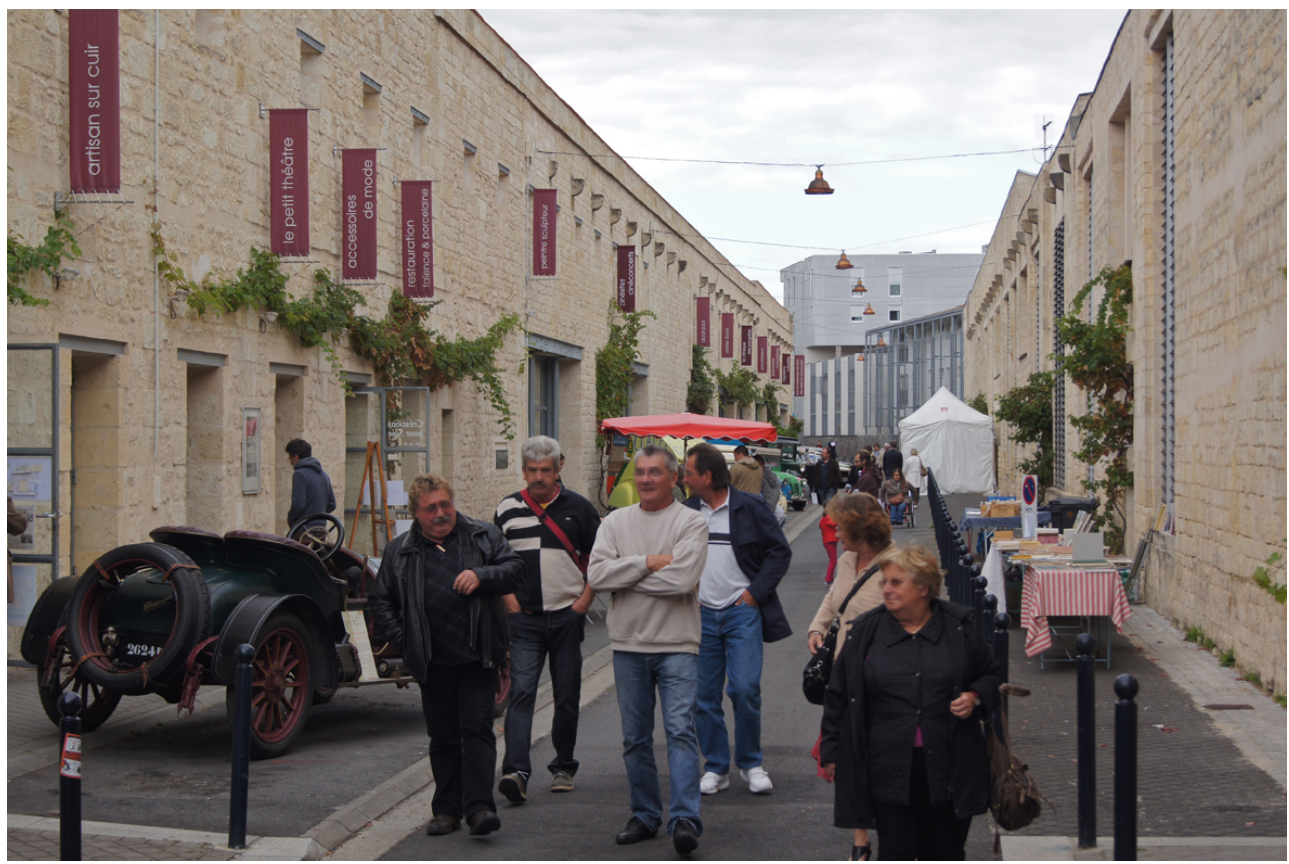

La rue du Faubourg-des-Arts un jour de fête.

Phot. Callais, Chantal. (c) Chantal Callais.

Pour offrir un rôle au nouveau quartier au-delà des activités quotidiennes, le projet originel prévoit un équipement exceptionnel, à l'échelle de la ville, qui permettrait de le relier aux quais des Chartrons. La vocation première des Chartrons incite à imaginer un grand équipement lié à la culture du vin: un Centre international du vin, juxtaposant « les aspects muséographiques touristiques culturels et professionnels liés au vin $»^{13}$.

On ne requalifiera globalement ce quartier qu'en revenant à ce qui en a fait la richesse... Les logements d'artistes, les ateliers, les artisans, les activités participent de la mixité urbaine mais, si ce centre ne se faisait pas, il y manquerait sans doute une valeur supplémentaire indissociable de ce quartier ${ }^{14}$,

31 affirme Jean-Luc Hoguet en 2000. Mais le grand programme de Centre du vin, situé sur les chais de Luze qui permettaient de le mettre en relation avec les quais par les immeubles des $n^{\circ s} 88$ et 89 , n'a pas passé le cap de la faisabilité économique et n'a pas vu pas le jour.

Alain Charrier avait imaginé dès la mise en place du plan d'aménagement que le principe d'alternance des lanières construites et libres autorisait facilement un changement de programme, soit vers un autre équipement, soit vers de nouveaux logements. Ouverte sur la rue Darbon, une partie de ce terrain accueille une pépinière écocréative pour de jeunes entreprises, ouverte en 2010. Les trois longues parcelles principales de la propriété de Luze ont permis d'ouvrir une galerie couverte distribuant des locaux à vocation diverse, activités commerciales ou publiques en rez-de-chaussée, administratives ou culturelles dans les étages (Michel Moga et Cécile Moga architectes). Elle permet de désenclaver le quartier qui souffrait d'être mal relié aux quais en cours de transformation par les grands aménagements effectués sous la direction du paysagiste Michel Corajoud. Des adaptations programmatiques affectent aussi d'autres lots : proche du gymnase, une lanière, d'abord destinée à un centre de formation ou à 
des locaux tertiaires, accueille une résidence-hôtel (Francis Martin architecte). Enfin, une belle voûte du XVII siècle, la voûte Schyler, dont on a découvert l'existence à l'occasion du projet de la ZAC dans la prolongation de celle des archives départementales, est d'abord acquise par la Ville qui la destine à un équipement de quartier. Achetée à la fin des années 1990, elle est revendue plus de six ans plus tard (2008) à des propriétaires privés qui ont l'intention d'en faire une galerie d'art et des ateliers d'artistes ${ }^{15}$, pour retourner finalement à sa vocation première, avec l'installation d'une entreprise de stockage de vins.

\section{De l'échelle urbaine à l'échelle architecturale}

Après l'établissement du projet urbain et de ses règles, plusieurs architectes (une dizaine) vont être invités à construire pour des maîtres d'ouvrage différents. Alain Charrier reçoit une mission de maîtrise d'œuvre urbaine pour le suivi et la coordination des projets d'architecture.

À Bordeaux, la méthode est nouvelle, assez inhabituelle pour les architectes bordelais : il faut nécessairement adhérer au principe de construire au sein d'un projet à l'échelle urbaine, qui impose des règles précises, mais adaptables, pourvu que chaque construction contribue à la qualité et à l'esprit de l'ensemble. Sur l'esthétique architecturale, peu de directives ont été données par Alain Charrier. Une seule exigence : aucun élément en saillie du volume de chaque bâtiment n'est admis; les volumétries simples sont alors considérées par l'architecte comme l'un des caractères définissant l'architecture contemporaine. Les architectes d'opération sont invités à "travailler en creux, à l'intérieur de l'alignement des façades " ${ }^{16}$. Si cette contrainte dérive d'une position personnelle de l'architecte et induit une formalisation sobre, elle constitue un facteur commun à toutes les opérations et elle a un impact sur le paysage urbain auquel il souhaite aboutir. Une maquette en image de synthèse insère les nouveaux projets au fur et à mesure de leur définition. Les différents concepteurs ont volontiers joué le jeu de contribuer à la formation du nouveau quartier en dialogue avec d'autres et ont apprécié d'avoir à « inventer dans un cadre prédéterminé », selon les termes d'Olivier Brochet, qui a dessiné l'un des immeubles d'habitation sur la place. La concertation qu'Alain Charrier instaure avec et entre les autres architectes est très organisée et régulière : des entretiens à plusieurs, entre les concepteurs de bâtiments mitoyens, et des rendez-vous individuels. Ainsi se mettent en place des éléments de cohérence formelle, tels des alignements de bandeaux, ou des harmonies de couleur, (voir fig. 24) sans pourtant aboutir à des écritures ou à des matériaux similaires. Un projet d'architecture peut même appuyer les options du projet urbain, comme le suggèrent les architectes Triaud et Arsène-Henry en proposant de composer l'immeuble qu'ils construisent sur le cours Balguerie-Stuttenberg en trois parties qui évoquent le rythme des trois « lanières » à l'arrière, alors qu'un volume unique avait été d'abord envisagé (fig. 18). 


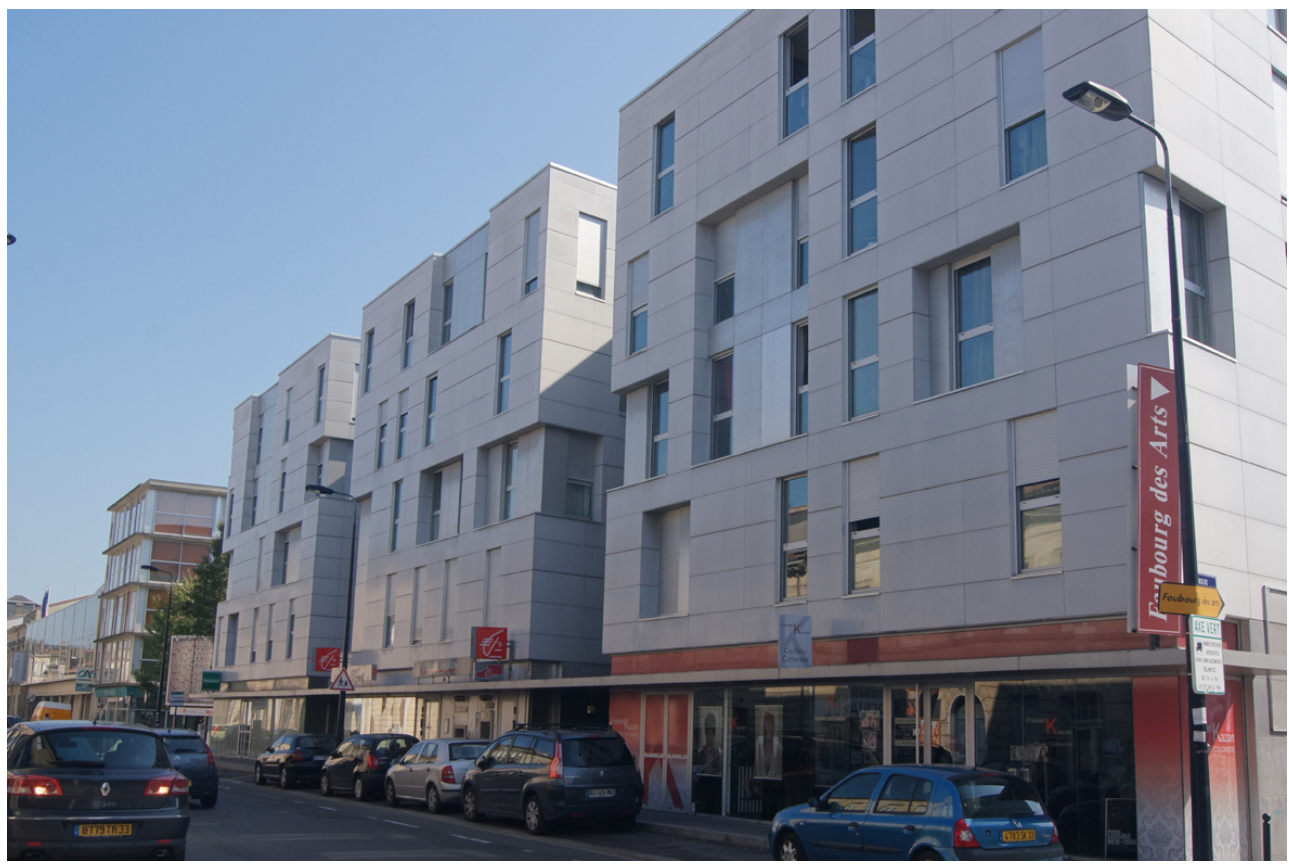

La partition en façade évoquant les trois lanières derrière l'immeuble du cours Balguerie-Stuttenberg (Triaud-Arsène-Henry).

Phot. Callais, Chantal. (c) Chantal Callais.

Pour les programmes d'habitat, de l'accession libre au locatif social, chaque architecte imagine des projets de logements différents selon les contraintes de la parcelle d'implantation. Un bâtiment-pont relié à un plot (Brochet-Lajus-Pueyo architectes) ferme une partie de la place (voir fig. 9), sur laquelle le jardin d'un "immeublesquare » s'ouvre dans la perspective du mail, plus largement que dans le schéma du PAZ (BBCR architectes) (voir fig. 14). Une lanière mono-orientée contre l'extension des archives départementales longe de ses redents le mail. Le dispositif mis en œuvre rappelle celui choisi par Yves Lion passage de Flandre à Paris dans les années 1980. Il permet d'offrir aux appartements des orientations diverses. La grande différence entre les deux opérations est le traitement des cours ainsi formées ouvrant sur la rue: passage de Flandre, elles permettent la plantation d'arbres qui scandent le paysage ; ici, c'est la voiture qui occupe tous les rez-de-chaussée. L'impossibilité de creuser les parkings à cette date a en effet rendu la présence de la voiture très prégnante dans la ZAC des Chartrons. Les stationnements sont parfois moins visibles, quand les parkings sont clos. En demi-sous-sol au centre du quartier, ils ont mis en commun les places de stationnement nécessaires à un ensemble de logements locatifs sociaux composé de deux opérations d'un même maître d'ouvrage : un immeuble collectif cours BalguerieStuttenberg (Triaud-Arsène-Henry) et à deux séries de maisons de ville sur le mail et la rue du Faubourg-des-Arts (Bühler architecte), ce qui permet de libérer une cour et une somme de terrasses privatives (voir fig. 15). À l'entrée de la rue Poyenne, un immeuble à jardin intérieur sur parking marque l'entrée dans le nouveau quartier du côté de la rue Poyenne (Loisier architecte). Le chantier de la ZAC s'est clôturé à la fin de l'année 2010 avec un immeuble d'habitation basse consommation rue Darbon (Cécile Moga architecte). Complétés par les équipements cités plus haut, le quartier accueille ainsi une grande variété de logements. 


\section{De la conservation aux écritures contemporaines}

Si le projet a misé beaucoup plus sur la structure parcellaire en lanières, très perceptible dans le paysage, que sur la conservation concrète d'objets architecturaux, il a permis la reconversion de plusieurs chais. Les quelques beaux entrepôts de la maison de Luze conservés offrent leurs murs de pierre dorée à la rue du Faubourg-des-Arts et à la grande galerie, le passage de Luze (fig. 19).

Figure 19

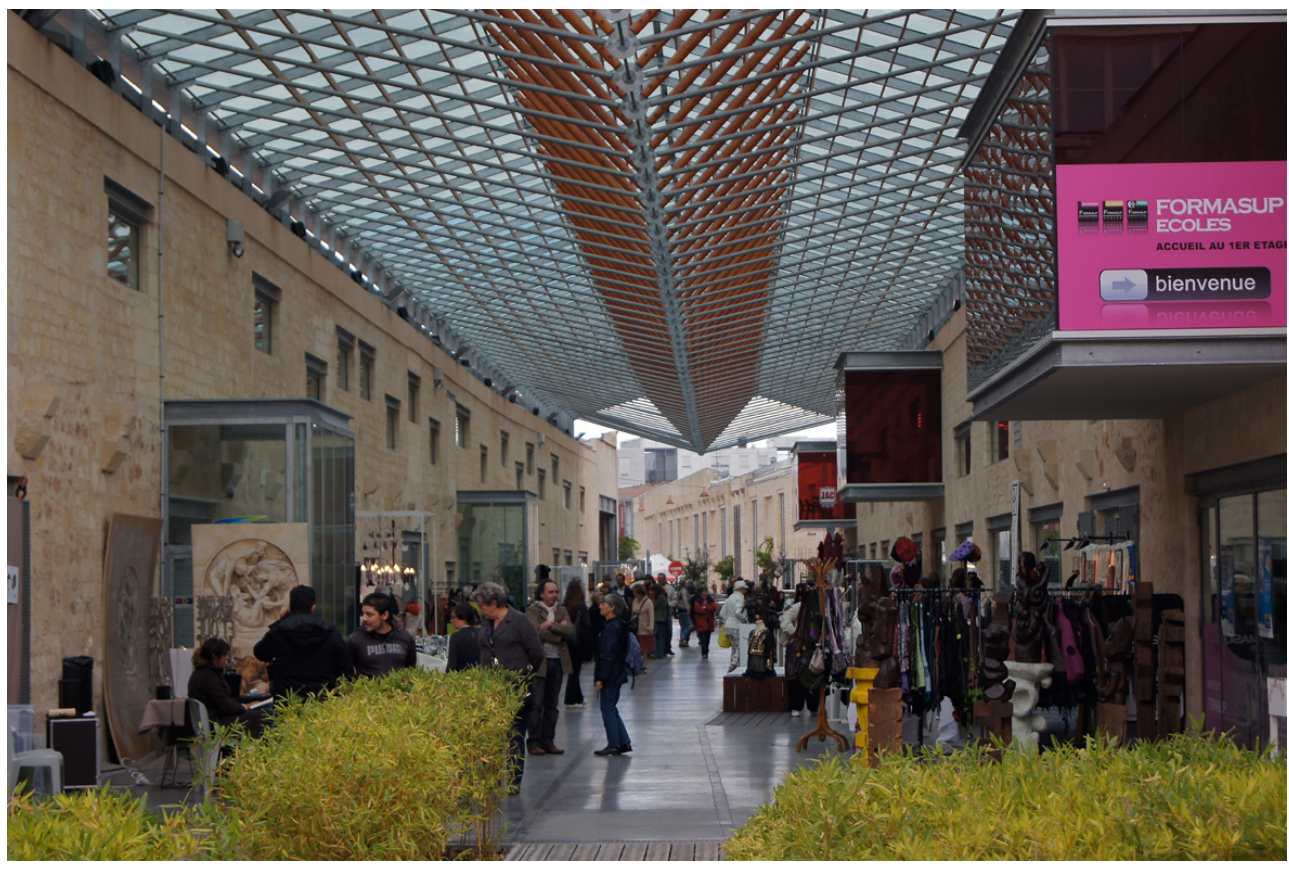

Le passage de Luze (Moga\&Moga architectes).

Phot. Callais, Chantal. ( C Chantal Callais.

37 Leurs murs ont été percés des ouvertures nécessaires pour l'école, les logements ou les ateliers-boutiques. Selon une méthode qu'il a souvent éprouvée dans des projets d'interprétation de chais en logements, Bernard Bühler double souvent l'ancien mur en pierre d'un mur moderne, soit pour offrir un espace d'entrée permettant d'établir une transition entre la rue et l'intérieur privé du logement (fig. 20), soit de créer une terrasse quand la maison n'a pas de jardin ou de cour. Des vignes plantées à leur pied offrent à la rue du Faubourg-des-Arts un paysage singulier par ce verdissement peu encombrant qui fait allusion aux activités anciennes du quartier. 


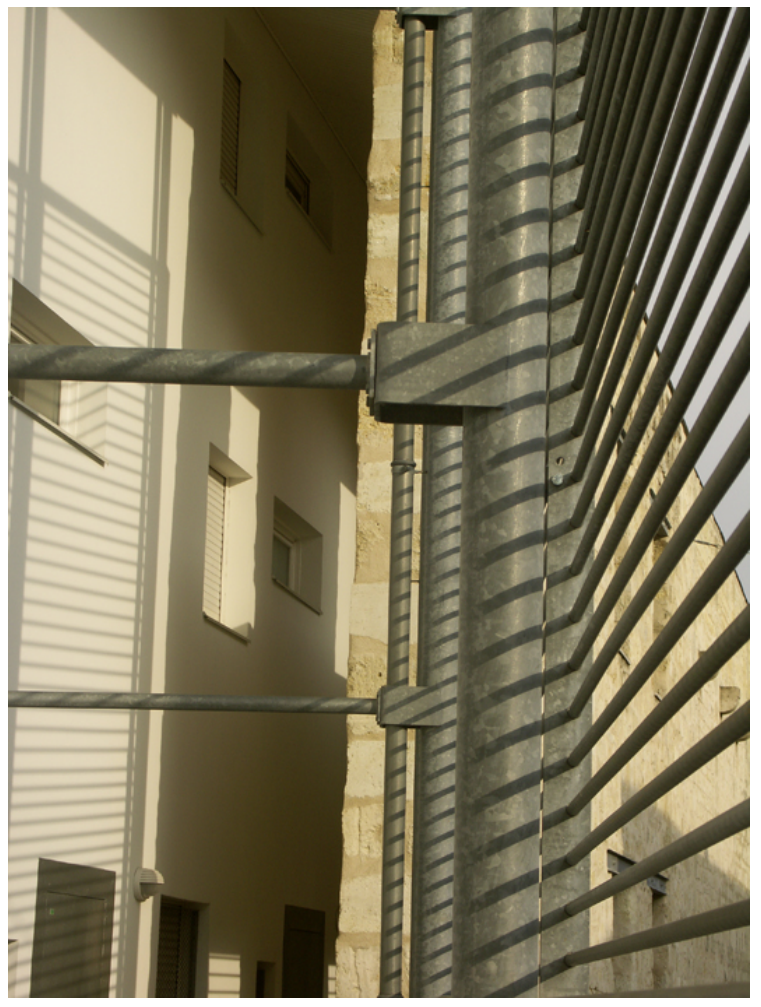

La double façade des maisons rue du Faubourg-des-Arts. Le mur de pierre de l'ancien chai est prolongé par une grille devant le mur des logements (B. Bühler architecte).

Phot. Callais, Chantal. (c) Chantal Callais. par une couverture en verre et métal. Ce clin d'œil au langage de l'architecture industrielle s'enrichit d'un dispositif de lames de bois qui filtre la lumière et souligne l'axe de la promenade couverte qui aboutit au passage sous immeuble permettant d'accéder aux quais. Cette traversée - la galerie 89 - par l'ancien courroir garde des traces de barriques sur l'un des murs et laisse apercevoir la magnifique cage d'escalier en pierre de l'un des immeubles de Luze (voir fig. 7). constructions entièrement nouvelles. Chaque maitre d'œuvre conserve une grande liberté de choix de ce point de vue. Outre les enveloppes des bâtiments aux volumétries simples, quelques détails révèlent discrètement le souci de dialogue établi entre les concepteurs sous l'égide de l'architecte-urbaniste: des ajustements de lignes horizontales, des harmonies de couleurs, entre des gris et des orangés plus ou moins lumineux... Une affirmation des performances de la rue comme espace fédérateur de diverses écritures architecturales, sans que le paysage urbain en paraisse incohérent.

\section{Le quartier aujourd'hui : atouts et faiblesses}

Après la douzaine d'années nécessaire à la mise en œuvre de ce projet (fig. 21), le quartier nouveau offre aujourd'hui un paysage urbain dans la continuité de l'existant, mêlant écritures contemporaines et interprétations des chais de pierre. Le respect de la singularité parcellaire du site a permis de proposer parfois des formes d'habitat 
atypiques qui participent à l'identité du lieu. Contre les idées parfois reçues du monde de la promotion, ce sont les logements les plus singuliers qui ont reçu la meilleure audience parmi les futurs habitants. La mixité fonctionnelle est riche, même si le grand programme de Centre du vin prévu au début n'a pas vu le jour : $28650 \mathrm{~m}^{2}$ de $\mathrm{SHON}^{17}$ de logements, $11425 \mathrm{~m}^{2}$ d'activités, bureaux et commerces et $5437 \mathrm{~m}^{2}$ d'équipements (école et gymnase).

Figure 21



Vue d'ensemble de la ZAC. À l'arrière de l'immeuble à redents, les archives départementales ne sont pas encore construites.

(c) J.-P. Boisseau/agence BBCR.

Le rôle majeur de Domofrance dans l'opération et sa maîtrise d'une grande partie du foncier a garanti une proportion importante de logements sociaux et en accession sociale $\left(244^{18}\right.$, auxquels s'ajoutent à la périphérie de la ZAC 74 logements réhabilités $\left.{ }^{19}\right)$, mais en mêlant ces programmes à des opérations privées, ce qui garantit une certaine mixité sociale. 

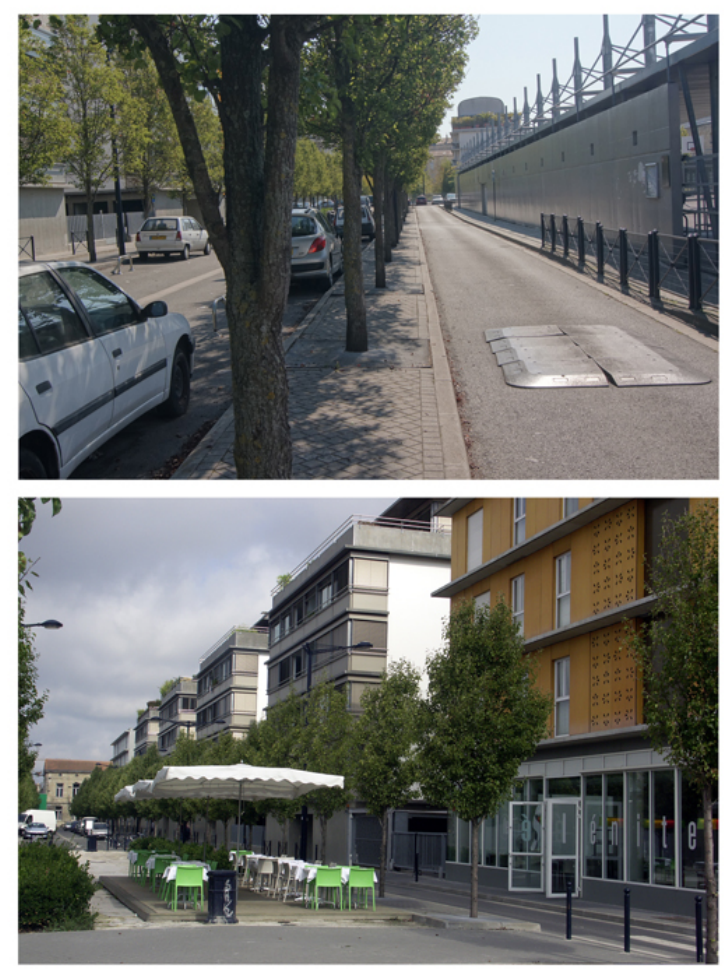

Vues du mail. L'aménagement laisse la plus grande place aux voitures ; seule une petite partie du terre-plein central, du côté de la place, est laissé à d'autres usages.

Phot. Callais, Chantal. (c) Chantal Callais.

Si densité et mixité répondent aux critères contemporains de la ville durable, la qualité des espaces publics du nouveau quartier est en grande partie pervertie par les stationnements automobiles. L'allée Stendhal comprend une partie consacrée à des parkings privés, qui détourne le principe d'aménagement d'un mail en supprimant l'espace de promenade qui le définit historiquement, réduisant l'espace laissé aux piétons à d'étroits trottoirs (fig. 22). Malgré quelques fauteuils installés de façon aléatoire, à la manière de certains aménagements à Barcelone, aux angles de la place (fig. 23), son paysage reste lui aussi très investi par l'automobile. À un moment où le grand projet de Bordeaux, lancé en 1996, avait comme objectif majeur le développement du tramway et son corollaire, la restitution des espaces publics aux piétons, la place abandonnée ici à la voiture peut étonner. Mais les esprits n'étaient pas encore prêts à imaginer des quartiers où l'accès de la voiture serait limité et des dispositifs nouveaux de parkings (comme des silos par exemple). Au-delà des espaces publics, de nombreux rez-de-chaussée sont aussi dédiés à la voiture, au détriment de relations directes des logements avec les jardins. 


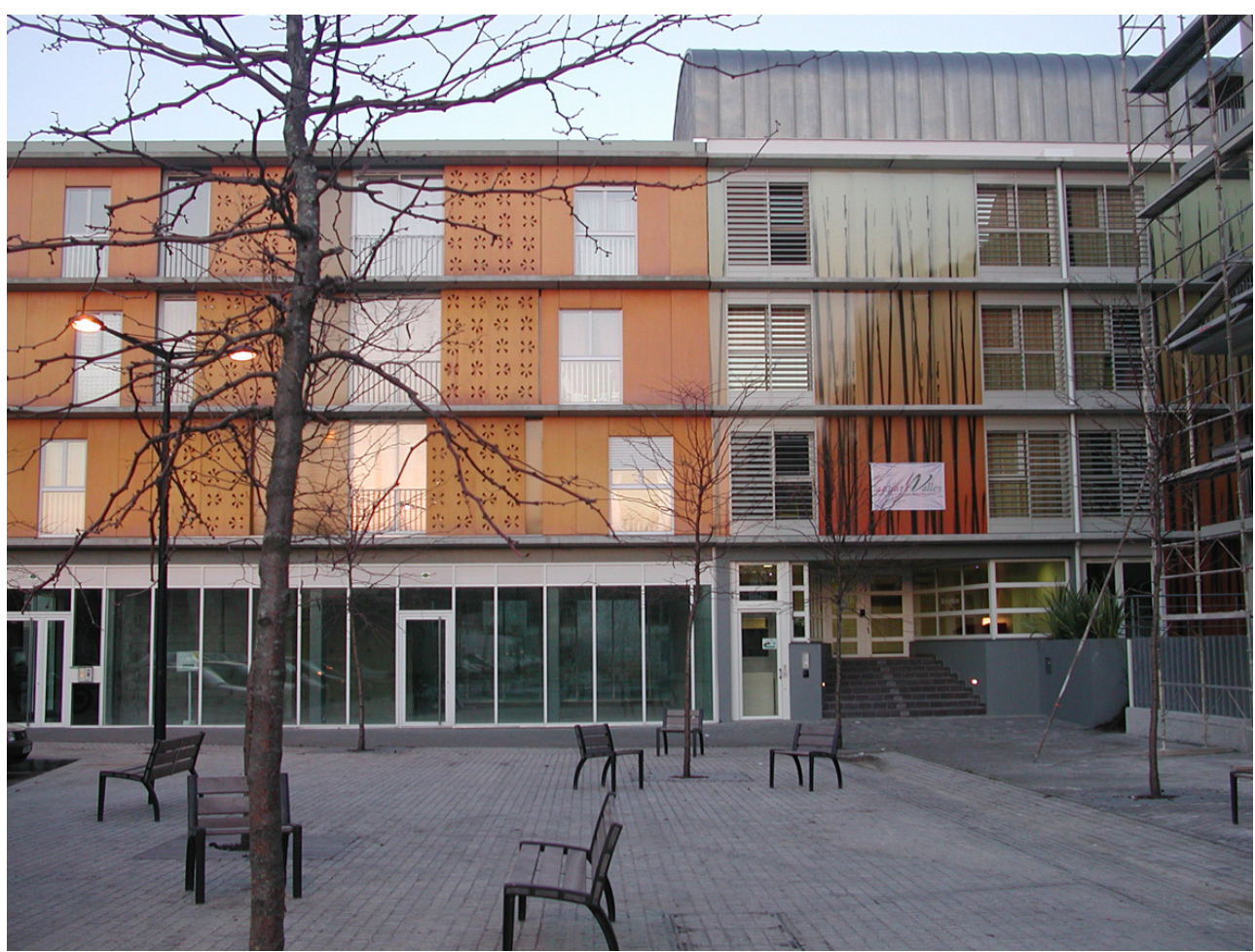

Aménagement des angles de la place. Les deux immeubles alignent leurs bandeaux horizontaux et harmonisent leurs couleurs.

Phot. Callais, Chantal. (c) Chantal Callais.

Si les architectes avaient prévu des lieux pour qu'une vie diversifiée s'installe, par la présence de boutiques et de commerces, le nouveau quartier a souffert du manque de liens directs avec les quais susceptibles de drainer, grâce au tramway et au hangar $14^{20}$, la population nécessaire au fonctionnement des commerces et restaurants. En 2007, un article du journal Sud-Ouest ${ }^{21}$ décrit les difficultés de la majorité des artistes et artisans, la déception des restaurateurs installés sur la place. Seuls les habitants sont contents, peut-on lire. Mais les politiques et les responsables de Domofrance ne se montrent pas très inquiets, considérant qu'il faut un temps pour que les activités commerciales s'installent dans un quartier nouveau. Il reste qu'à cette date les deux opérations comprenant une ouverture vers les quais, le gymnase et le passage sous l'immeuble du $\mathrm{n}^{\circ} 89$ dans la continuité du passage de Luze ne sont pas terminés. Deux ans plus tard, en juillet 2009, la galerie 89 n'est toujours pas achevée et la mairie, sensible aux demandes des habitants, demande que le propriétaire ouvre ce passage pendant un créneau le plus large possible de façon à ce que les enfants puissent emprunter ce "passage sécurisé22 ». En effet, la petite rue Barreyre, peu empruntée, n'est pas très avenante et le projet d'en élargir les trottoirs, annoncé par l'adjoint au maire en 2007, n'a toujours pas vu le jour ${ }^{23}$.

Sans en être l'unique raison, il apparaît que le désenclavement de la zone par l'ouverture de la galerie sur les quais a certainement été un élément catalyseur pour qu'une vie qui apparaît aujourd'hui de plus en plus foisonnante s'installe dans la ZAC, acronyme devenu ici quasiment un nom propre, emprunté par un restaurant dont les prix sont accessibles aux étudiants (la «ZAC» pour «zone d'activité culinaire»). La nécessité de pouvoir accéder au quartier depuis les quais est confirmée par la 
fréquentation du passage longeant le gymnase (fig. 24), qui n'est inauguré qu'en 2009 : la structure en bois de la grille qui le ferme a été soigneusement partiellement démontée de façon à laisser un passage libre en permanence et la grille du côté des quais n'est pas toujours fermée.

Figure 24

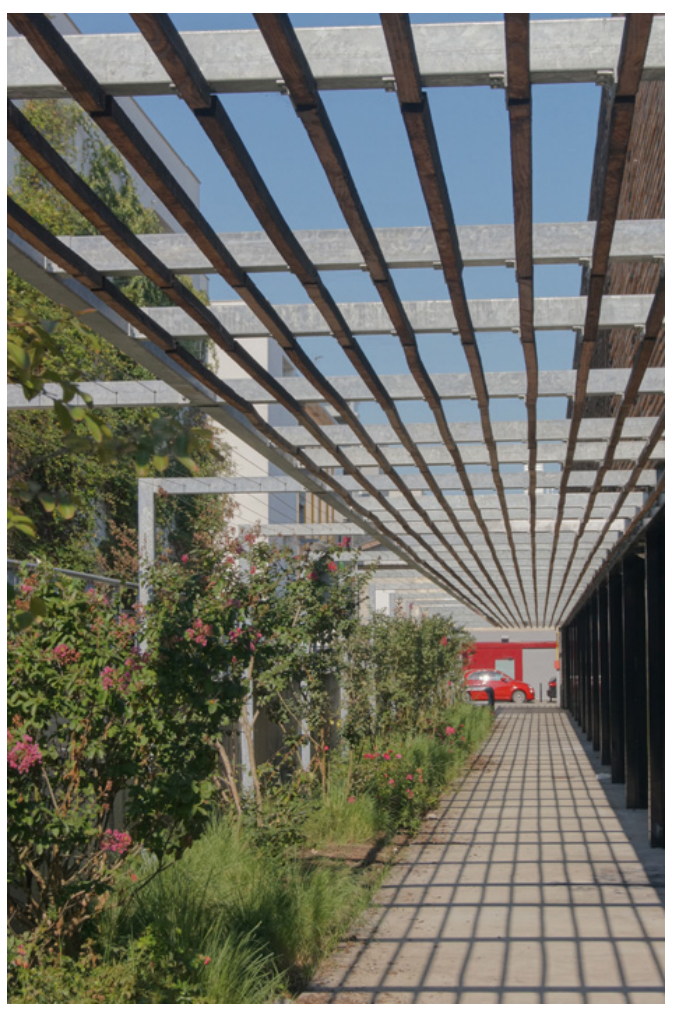

Le passage longeant le gymnase (Baudin\&Limouzin architectes)

Phot. Callais, Chantal. ( ) Chantal Callais.

Conserver la dimension introvertie du quartier a été une volonté du projet dès l'origine. Aurait-il fallu ouvrir plus tôt les liaisons depuis les quais? Un lien plus évident et plus direct avec la rue Notre-Dame et ses activités commerçantes aurait-il accéléré le processus de mise en place des activités ? Il n'est pas possible de l'affirmer, tant les phénomènes urbains sont complexes et font entrer en jeu une multitude d'acteurs et de critères.

Il est certain qu'au-delà du passage qu'elle permet (et bien qu'elle reste fermée les jours fériés, devenant alors imperceptible depuis les quais (fig. 25), la grande galerie a été un facteur important du "démarrage " réel du quartier. Elle apporte une population nouvelle et mêlée, des lieux de formation, des professions libérales et divers bureaux ${ }^{24}$ qui permettent de faire vivre plusieurs restaurants aujourd'hui installés autour de la place, après le renoncement des premiers installés ici. Le passage de Luze offre aussi un espace rare et séduisant, à la fois monumental et convivial, fermé et ouvert, capable de fédérer multiples activités, de la déambulation à la pause-discussion ou restauration. Avec la rue du Faubourg-des-Arts, c'est le lieu-phare de la ZAC, tandis que l'allée Stendhal pâtit de sa vocation de parking et des nombreux rez-de-chaussée aveugles qui la bordent. 
Figure 25



Les trois immeubles de Luze. L'entrée de la galerie 89 se situe sur l'immeuble du centre.

Phot. Callais, Chantal. (c) Chantal Callais.

Aujourd'hui, et depuis peu de temps, avec la fin des travaux et l'occupation complète du site (en 2011, le nouveau bâtiment des archives départementales mitoyen de la ZAC est lui aussi inauguré), il apparait que tous les ingrédients sont réunis pour faire de la ZAC des Chartrons un des quartiers vivants du nord de Bordeaux. Si l'espace et la forme ne font pas tout, ils apparaissent comme les supports potentiels et essentiels aux activités. Les espaces publics qui ont été dessinés ici contribuent largement à tisser la ville et à y intégrer ce nouveau quartier, en lui offrant pourtant une ambiance singulière par le dialogue instauré entre les traces de son histoire, une interprétation moderne de la ville classique, notamment par les «transparences" entre l'espace public et les espaces privés, et enfin des écritures architecturales contemporaines. Inscrite dans ces espaces, la "ZAC » a pris son envol avec une vie de quartier animée, qualifiée « d'esprit de village » par un article de Sud-Ouest ${ }^{25}$.

Les artistes et artisans installés dans la rue du Faubourg-des-Arts, où ils bénéficient de loyers modestes pour un atelier voûté et un logement au-dessus, se sont longtemps plaints de leur isolement et du manque de chalands. Pour exploiter l'atout d'être regroupés, ils se sont fédérés il y a environ six ans au sein de l'association des « arts pas sages ", qui organise des manifestations destinées à les faire connaître. Sans renoncer aux activités partagées ${ }^{26}$, le manque de liaison avec la rue Notre-Dame est oublié au profit d'une mise en valeur de cette rue qui bénéficie de sa continuité avec les quais par le passage de Luze et la galerie 89. Le patron de l'un des restaurants de la place apprécie "le mélange des classes sociales [qui] fait bouger le secteur dans le bon sens, même s'il se " boboïse " un peu ${ }^{27}$ ». L'association « Esprit de quartier » contribue à l'affirmation de son identité. Organisant cinéma en plein air et vide-greniers, elle a le projet, de connivence avec la maire adjointe de ce quartier, de limiter l'emprise de la voiture sur 
la place pour la rendre aux arbres et aux hommes et d'installer des jardins partagés sur une friche proche, sur la rue Barreyre ${ }^{28}$. Toute cette activité attire de nouveaux arrivants dans des locaux aujourd'hui convoités par d'autres artistes et des professions libérales.

La ZAC des Chartrons a initié à Bordeaux les pratiques de renouvellement urbain et de projets cherchant une identité propre dans leur ancrage dans l'histoire et l'exploitation du génie de chaque site, qui se sont développées depuis et incluent maintenant dans leurs objectifs ceux de la ville durable. De nombreux points de vue, la ZAC des Chartrons y répondait déjà, son principal défaut étant la place qu'elle attribue à la voiture. Mais, après le projet évoqué pour la place Paul-et-Jean-Paul-Avisseau, il n'est pas impossible d'imaginer que l'allée Stendhal soit un jour réaménagée au profit d'une promenade plantée pour faire évoluer la ZAC vers un «écoquartier ».

\section{NOTES}

1. - LIQUARD, A. Les chais des Chartrons à Bordeaux. Mémoire sous la dir. de David Mangin, ENSA Versailles, 1987 p. 16. Ce travail présente l'analyse la plus complète aujourd'hui de la typologie architecturale des Chartrons.

2. - Ibid.

3. - Cité par LIQUARD, A., ibid.

4. - PREVOT, Ph., ZEBOULON, R. Bordeaux secret et insolite. Paris : Les beaux jours, 2005, p. 120.

5. - LIQUARD, A., op. cit., p. 35.

6. - Ibid., p. 19-20.

7. - Ibid., p. 24.

8. - Ibid., p. 50.

9. - «Bordeaux-Les Chartrons. Une transformation sans a priori ». Projet urbain, ${ }^{\circ} 19$, mars 2000 , p. 7-10.

10. - Voir ROSSI, Aldo. L'architecture de la ville. Gollion, In folio, 2001 (traduction française du livre paru en italien en 1960).

11. - DUBOURG, S. (propos recueillis par). «Alain Charrier, architecte-urbaniste, l'îlot des Chartrons ». Art dans vivre, ${ }^{\circ} 5$, mars 1995, p. 25-27.

12. - L'analyse du projet et de sa genèse est fondée sur les conférences données par Alain Charrier sur sa démarche et sur un mémoire d'Élodie Martin qui relate le processus du projet à partir d'entretiens avec l'architecte-urbaniste et des archives de son agence (Le patrimoine au service de la modernité, ENSAP Bordeaux, sous la direction de Chantal Callais, 2002).

13. - Propos de Jean-Luc Hoguet, Projet urbain, art. cit., p. 9.

14. - Ibid.

15. - Délibération du conseil municipal de Bordeaux, 16 juin 2008, en ligne.

16. - Projet urbain, art. cit., p. 8.

17. - Surface hors œuvre nette.

18. - Dont 125 en PLA/PLUS, 19 en PLI et 12 en PCL, 68 en accession (Renouvellement urbain, «L'îlot des Chartrons à Bordeaux-Domofrance ", en ligne).

19. - Dont 62 en PLA et 12 en PLUS. 
20. - Premier de ceux conservés sur les quais, le Hangar 14 a été réhabilité en lieu d'expositions et de manifestations.

21. - MATHURIN, Hervé. « Ouvrir le cul-de-sac». Sud-Ouest, 7 août 2007.

22. - Direction générale de la Vie urbaine et de la Proximité, direction de la Vie locale, service Vie des quartiers, compte rendu de la réunion d'information relative à l'aménagement de la ZAC des Chartrons : présentation du projet du groupe Réaumur, mardi 7 juillet 2009.

23. - MATHURIN, H., art. cit.

24. - Dont Réseau ferré de France, le consulat des États-Unis, des écoles de formation.

25. - MANENC, Dominique. « L'esprit de village revient ». Sud-Ouest, 7 octobre 2011.

26. - L'association "Arts Chartrons » par exemple, née en 2007, organisa en novembre 2011 la septième édition des parcours d'art contemporain.

27. - Cité par MANENC, Dominique, art. cit.

28. - Ibid.

\section{RÉSUMÉS}

Le quartier du négoce bordelais, les Chartrons, se compose de l'assemblage dense d'un type architectural qui combinait la partie noble et la partie fonctionnelle des entreprises, les chais. Le quartier est tombé en désuétude pendant la première moitié $\mathrm{du} \mathrm{xx}^{\mathrm{e}}$ siècle, laissant les entrepôts libres de toute occupation. Depuis le milieu des années 1980, ce quartier trouve une nouvelle vie grâce au remploi des longs chais mitoyens. Un îlot exceptionnellement vaste a fait l'objet d'une reconversion depuis 1993. Ce projet présente l'intérêt d'agir à deux échelles, celle de la ville et celle de l'architecture, qui inscrivent le quartier nouveau dans une filiation historique.

The Chartrons neighbourhood of Bordeaux was traditionally occupied by wine merchants, and has a specific urban form of densely packed buildings called 'chais', wine cellars, which combine the noble commercial activities of the merchants and their more functional storage ones in single buildings. During the first half of the twentieth century, the neighbourhood went into decline, leaving many of the warehouses without any use. Since the mid-1980s, however, the neighbourhood is finding new life, thanks to the re-use of these long, neighbouring buildings. Since 1993 one particularly large area known as the 'grand îlot' has undergone an interesting operation of reconversion, operating at two levels, that of the city as a whole and that of the individual buildings, giving this 'new' neighbourhood its historical filiation.

\section{INDEX}

Keywords : reconversion of industrial premises, heritage, Bordeaux, Chartrons, urban projects, 'chais', wine cellars

Mots-clés : reconversion locaux industriels, patrimoine, Bordeaux, Chartrons, projet urbain, chais 
AUTEUR

\section{CHANTAL CALLAIS}

architecte-urbaniste, docteur en histoire de l'architecture, équipe de recherche GEVR/ADES-UMR 5185-CNRS - ENSAP Bordeaux/Bordeaux Montaigne chantal.callais@bordeaux.archi.fr 